\title{
Transition of Space Propulsion and Challenge to the Future -Breakthrough of Propulsion Technology
}

\author{
Yoshinari Minami \\ Advanced Space Propulsion Investigation Laboratory (ASPIL) \\ (Formerly NEC Space Development Division), Japan
}

Received: July 24, 2019 Accepted: August 16, 2019 Published: August 18, 2019

Doi: 10.5296/ijca.v3i1.15289 URL: http://doi.org/10.5296/ijca.v3i1.15289

\begin{abstract}
The distance to a stellar system is very immense, therefore the travel to the fixed star nearest to the Earth using the present propulsion technology will require tens of thousands years. In order to overcome such a limit of the space travel between fixed stars, research and development of a new propulsion theory and navigation theory are indispensable.

This paper describes the historical transition and future prospects of space propulsion technology, that is, new space propulsion and space navigation concept (Laser thruster, Continuum mechanics of Space-Time, Space drive propulsion, Hyper-Space navigation etc.) based on the published article so far by author. The prospects of various applications of laser thruster, ion thruster as electric propulsion and solar sail are introduced. A field propulsion which surpasses conventional propulsion is then discussed as well as the possibility of realistic interstellar travel by applying field propulsion to navigation theory.

Space propulsion physics such as propulsion theory and navigation theory give us a concrete theoretical method toward galaxy exploration.

Keywords: Space propulsion, Field propulsion, Space drive, Laser thruster, Interstellar travel, Hyper-Space navigation, Time-hole, Wormhole, Starship

\section{Introduction}

For more than one hundred years since the World's first powered flight by the Wright Brothers in 1903 (Figure 1), power and propulsion for air and space travel has been the explosive combustion and heating of matter in a chamber and expulsion of its hot gases. But this may eventually end. As such consuming, heating, and emitting of matter cannot propel space-ships swiftly enough or far enough to go where much of humanity wants to go.
\end{abstract}




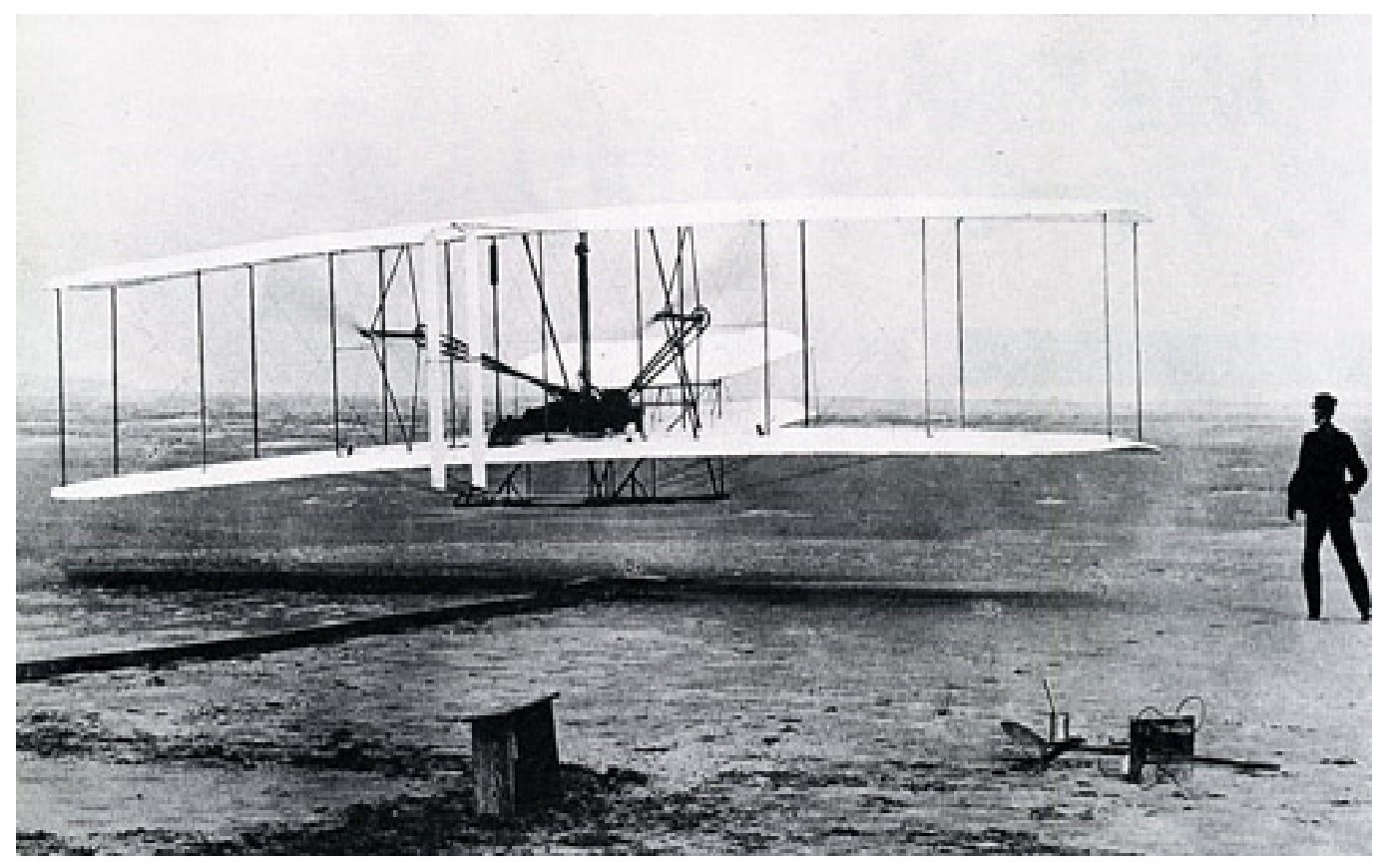

Figure 1. World's first powered flight by the Wright Brothers

In the case of space travel, combustion-heating-expulsion takes place in the chambers of solid or liquid propellant chemical rockets for almost 90 years since Robert Goddard's first chemical liquid rocket launch in 1928. But now, almost 90 years later, chemical rockets have reached a plateau in the thrusting performance that they can achieve. And though remarkable, it is woefully insufficient for propelling spaceships economically, swiftly, safely over the enormously long gulfs of inter-planetary and interstellar space that much of humanity dreams of going. Also, the consuming-heating and emitting of gases, which has met Earth's terrestrial power and propulsion needs for over 150 years is now heating its atmosphere at unacceptable rate.

The dream of space travel by rocket propulsion that originated the edge in 1903 has steadily expanded the range of the exploration to not only the moon but also other planets in the solar system by the predecessors' efforts. As is well known, chemical rocket propulsion system, ion propulsion engine of "Hayabusa (MUSES-C)" (Kuninaka, 2006) and solar sail "IKAROS (Interplanetary Kite-craft Accelerated by Radiation of the Sun)" support this technology of space exploration at present.

It is a beginning the landing on the moon by Apollo 11 on July 21, 1969, that manned space exploration by the human was performed. The arrival time to the moon depends on the selected orbit; for example in the case of Apollo 12, it took three days (for the way home 72 hours for outward trip 80 hours). Such small amount of navigation time (3 days) is not the problem, but in the case of a more distant remote planet from the Earth, it is an already dreamlike story because the speed is late in the current rocket technology.

The distance $384,400 \mathrm{~km}$ (cislunar distance) is too short distances as seen from a universe scale. It can be said that traveling to the planet and the stars by the method of going to the moon is impossible due to the present rocket technical limitation. It is a fact that mankind has 
no possession of the space propulsion technology that can accelerate at a high speed in a short time. Comparing space as the ocean, mankind is obtaining only a technology moved in the vicinity of the shore, as moving near sand beach with a boat. It is only the moon that mankind could reach. Apart from the unmanned spacecraft, mankind has a boat that goes near the beach, and we do not yet have a ship that is going through the vast universe. If the speed of spaceship increases marvelously, that is, if the arrival time to the planet is becoming several hours or several weeks, it is considerably surmountable. It is understood that the development of a space transportation system of the very high speed is important for the manned space exploration.

At the present stage of space propulsion technology, the only practical propulsion system is a chemical propulsion system and an electric propulsion system, which are based on the expulsion of a mass to induce a momentum thrust. Since the maximum speed is limited by the product of the gas effective exhaust velocity and the natural logarithm of mass ratio, its speed is too slow for the spaceship to achieve interplanetary travel and interstellar travel. Thus, the breakthrough of propulsion method has been required until now.

Instead of conventional chemical propulsion systems, field propulsion systems, which are based on General Relativity Theory, Quantum Field Theory and other exotic theories, have been proposed by many researchers to overcome the speed limit of the conventional space rocket. Field propulsion system is the concept of propulsion theory of spaceship not based on momentum thrust but based on pressure thrust derived from an interaction of the spaceship with external fields. Field propulsion system is propelled without mass expulsion. The propulsive force is a pressure thrust which arises from the interaction of space-time around the spaceship and the spaceship itself; the spaceship is propelled against space-time structure.

So author believes now is the time to begin serious exploration of a nearly propellant-less mode of power and propulsion - one that creates energy, power, thrust by actions and reactions of mass-less fields - not by combustion and emission of mass.

At the end of this chapter as a note, this is a brief introduction to Ninomiya Chūhachi, who first discovered the theory of fixed wings and flight principles in Japan ("Crow-type model aircraft", 1891) (http://hikoujinjya.kyoto.jp/yuisho.html). He designed a flying machine with three engines earlier than the Wright brothers, and, even though the machine failed to take-off, it contributed to Japan's accumulation of capabilities to design and manufacture unique world-class aircraft by the 1930s. He retired from the army and went work at a pharmaceutical company. He then decided to develop a flying machine on his own.

During this period, the Wright brothers succeeded with a manned flight. But Chūhachi did not know the news. He built the whole hull of the "Tamamushi model" and planned to equip it with a $12 \mathrm{hp}$ gasoline engine. However, as the Wright brothers of the United States completed the plane on December 17, 1903, he learned of the success of manned flights of heavier-than-air aircraft later. He became despaired and stopped his development. The reason of cessation of production was due to "Now, even if I made an airplane, it would only receive an evaluation that it resembled Wright brothers."

All are the history of humanity's challenge for the dream towards the sky.

In the following chapters, we introduce star flight by Field Propulsion. 


\section{Space Travel by Field Propulsion}

\subsection{Technical Status and Problem of Present Propulsion System}

The problems of existing space propulsion system are introduced in this section.

\subsubsection{Overview of Conventional Propulsion Technologies}

At the present day, aside from the practical chemical propulsion system, many propulsion systems and concepts are investigated. Those are as follows: Electric propulsion system (ION thruster, MPD thruster, ARC jet thruster, and Hall thruster), Laser propulsion system, Nuclear propulsion system, Solar sail, Light sail (Niino, Minami, \& Nakamura, 1997). Figure 2 shows an example of ion thruster.

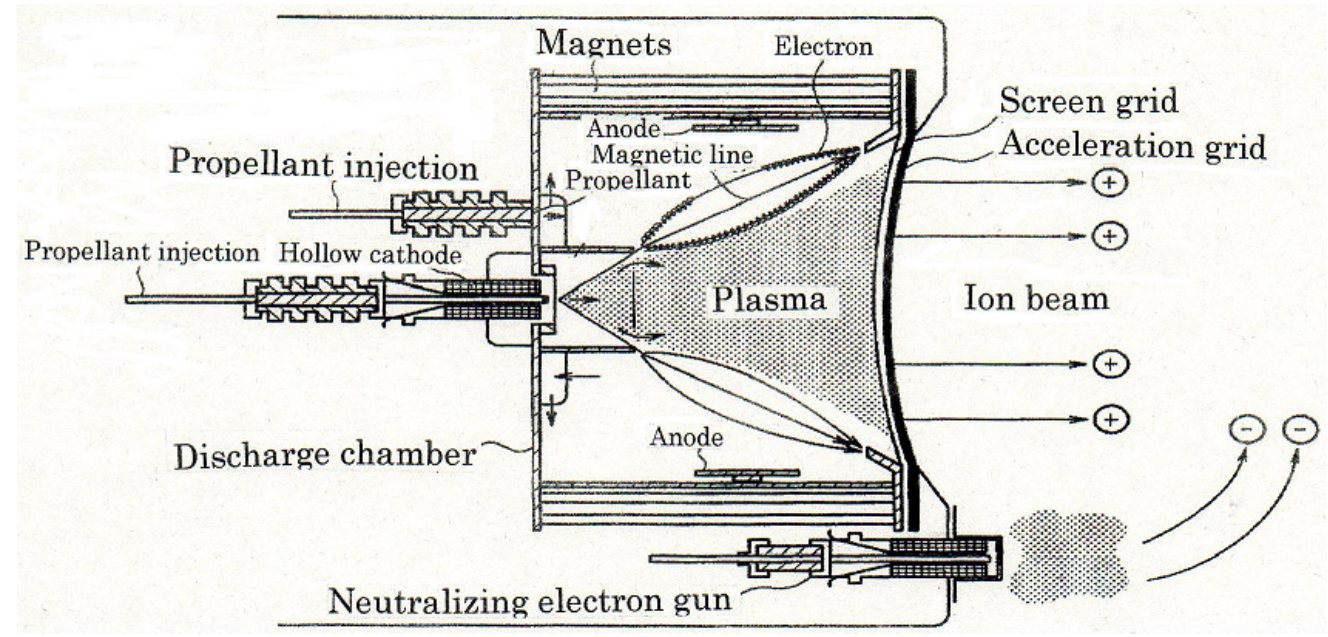

Figure 2. Kaufman type ion thruster

All kinds of current propulsion system except solar sail or light sail are based on the momentum conservation law. In the case of the momentum thrust based on momentum conservation law, the maximum speed (V) is limited by the product of the gas effective exhaust speed $(\mathrm{w})$ and the natural logarithm of mass ratio $(\mathrm{R})$.

$$
V=w \cdot \ln R=g I_{S P} \cdot \ln R
$$

The maximum speed $\mathrm{V}$ which a rocket can reach is theoretically determined by the gas jet speed $\mathrm{w}(\mathrm{m} / \mathrm{s})$ and the mass ratio $\mathrm{R}$.

Because the velocity of present rocket is too slow as compared with the speed of planet, the interplanetary exploration by Mankind, not to speak of interstellar exploration, has various technical difficulties. We need the super-high speed and high acceleration of spaceship.

For example, the origin of the problem that the manned Mars exploration takes long-term time is due to the cruise velocity of a spaceship being too slow. The second astronomical speed $(11.2 \mathrm{~km} / \mathrm{s})$ that a rocket obtains for earth escape is slightly slow compared with the orbital speed $(24 \mathrm{~km} / \mathrm{s})$ of the Mars or the orbital speed $(30 \mathrm{~km} / \mathrm{s})$ of the Earth.

This is because the maximum speed of a rocket is limited by the product of gas effective 
exhaust speed and the natural logarithm of mass ratio (its value is about 7). The speed beyond this cannot be theoretically taken out from the propulsion principle of a rocket based on the momentum conservation law.

Concerning a chemical rocket which has multi-stage composition, about $10 \mathrm{~km} / \mathrm{s}$ speed is the practical limit. In the case of a chemical rocket, its $I_{S P}$ is 460 seconds, so the maximum speed becomes $4.5 \mathrm{~km} / \mathrm{s}$ for single stage rocket. If the speed of a rocket is 1000 times quicker compared with the speed of the Mars or the Earth, a straight line trajectory can be attained. Whenever you wish, it can reach to the target planet always in a short time without restriction of orbital calculation, start time and return time, so that it may operate as if it were a automobile.

Equation (1) can be represented as follows in detail:

$$
V_{f}-V_{i}=\Delta V=\int_{0}^{T} \alpha d t=\int_{0}^{T} \frac{F}{m} d t=\int_{0}^{T} \frac{I_{S P}(-\dot{m} g)}{m} d t=I_{S P} g \ln \frac{m_{i}}{m_{f}} .
$$

Equation (2) indicates the speed increment $\Delta V$ of a rocket when the rocket of the initial mass $m_{i}$ reduces mass to the rocket of the final mass $m_{f}$ by combustion for T seconds. Since the propellant mass $m_{p}$ is given by Equation (3), combining Equations (2) and (3) yields the Equation (4):

$$
\begin{gathered}
m_{p}=m_{i}-m_{f} \\
m_{p}=m_{i}\left[1-\exp \left(-\frac{\Delta V}{g I_{S P}}\right)\right] .
\end{gathered}
$$

By expelling the mass of a propellant $m_{p}$ outside, the rocket obtains and increases speed $\Delta V$, that is, the propellant is indispensable for the conventional propulsion system based on the momentum thrust. Further, since a large thrust is required for the large weight of payload, a large amount of propellant is needed for the rocket; therefore the increased weight of the rocket needs more larger amount of propellant.

From such a situation, NASA established the research project named "Breakthrough Propulsion Physics (BPP) project" in December, 1996.

In the latest scientific research paper published, a new theory and a new phenomenon are discovered and the possibility of a space transportation system of a certain kind which can convey human beings to other star systems is suggested. The greatest concern of NASA's BPP research is the theory and the basic experiment about combination of gravity and an electromagnetic field, fluctuation of vacuum zero-point energy, a warp drive, a wormhole, the quantum gravity effect, etc. In order to overcome such a limit of the space travel between fixed stars, research and development of a new propulsion theory, propulsion engineering, and navigation theory are pursued by this project. 
Accordingly, we need new propulsion principle to exceed the limits of prior propulsion technology and seek entirely different technology.

As described in NASA BPP, we must investigate the new propulsion system based on physics as follows.

1). Mass: Discover new propulsion methods that eliminate the need for propellant or beamed energy.

2). Speed: Discover how to circumvent existing limits (light-speed) to dramatically reduce transit times.

3). Energy: Discover new energy methods to power these propulsion systems.

\subsubsection{Pressure Thrust}

As compared with momentum thrust, pressure thrust principle is to be pushed from the back and thus move forward. Solar sails or Light sails use this principle (Figure 3).

Pressure thrust also partly contributes to rocket and jet aircraft. That is, since the engine nozzle pressure at the rear of the rocket is larger than the atmospheric pressure at the front of the rocket, the rocket is pushed out from behind. As another example, the swimmer turns to push the wall of the pool with a foot; a car tire pushes the ground of the Earth, etc.

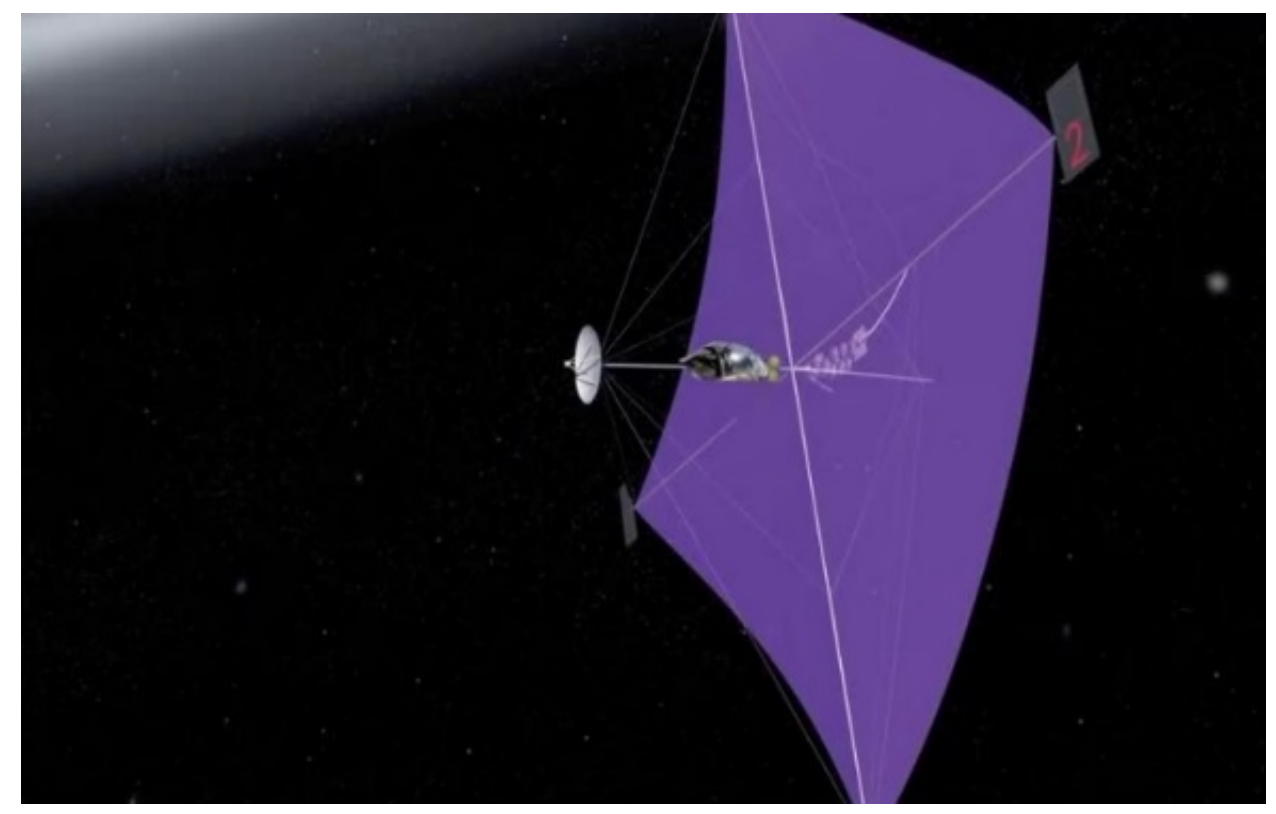

Figure 3. Solar Sail, Light Sail 


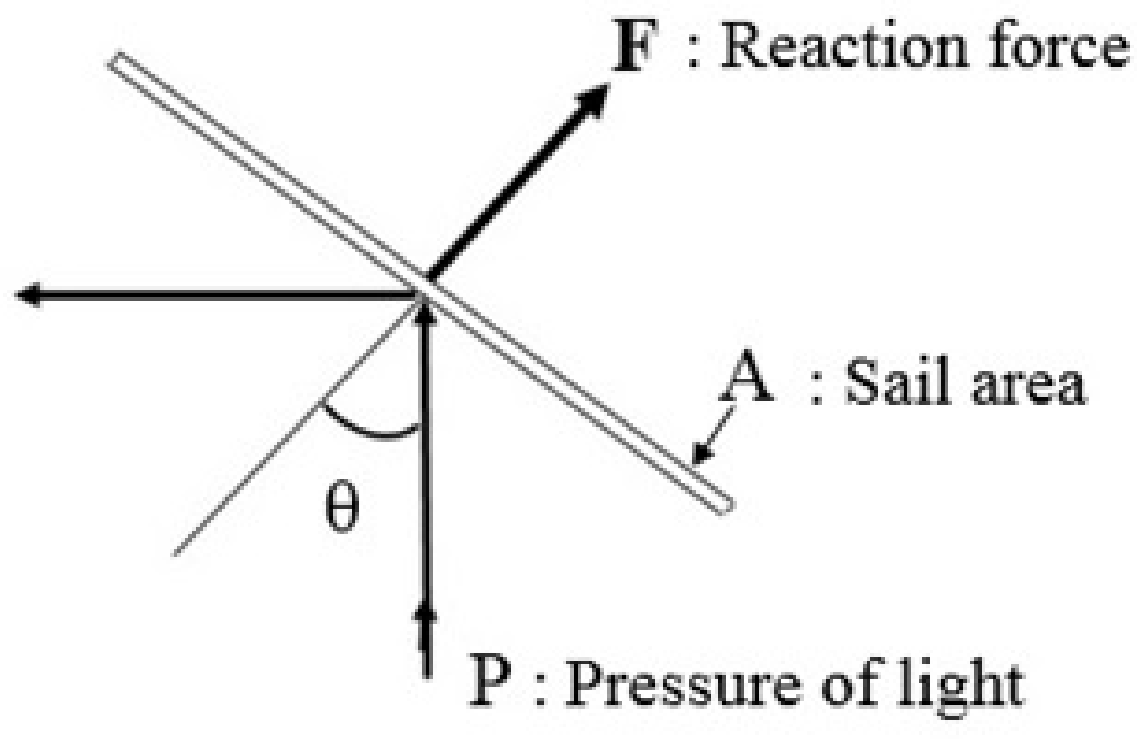

Figure 4. Reaction force of light received by sail

As shown in Figure 3, the solar sail or light sail spreads large lightweight sail in outer space and propels by receiving light pressure as wind. It will be pushed and accelerated by light pressure, so there is no limitation of speed, i.e., the maximum final speed is close to the light speed (quasi-light speed). However, since the light pressure is very small, the thrust is small, and the acceleration is a very small value of several micro G. If it is 1 micro G acceleration, it will take about 3 years to reach $1 \mathrm{~km}$ per second.

To reach $100 \mathrm{~km}$ per second, it has to be kept pushed for about 300 years. But if we only have time to spare, we can reach the speed close to the light. Somehow, we want to get a large acceleration of a few $\mathrm{G}$.

Referring to Figure 4, the reaction force of light received by sail is given by

$$
F=P_{p} A(1+\varepsilon) \cos \theta
$$

where $F$ : reaction force $(\mathrm{N}), \mathrm{P}_{\mathrm{p}}$ : the pressure of light $\left(\mathrm{N} / \mathrm{m}^{2}\right), A$ : sail area $\left(\mathrm{m}^{2}\right)$, $\varepsilon$ : reflectance of light.

In case that the sail is perpendicular to light $(\theta=0)$ and perfect reflection $(\varepsilon=1)$, we get

$$
F=2 P_{p} A
$$

Solar light pressure near the Earth is very small $\sim 4.6 \times 10^{-6} \mathrm{~N} / \mathrm{m}^{2}$. Assuming that the area density $\beta$ of the sail is about $\beta=100 \mathrm{~g} / \mathrm{m}^{2}$, the acceleration $\alpha$ of the sail is, $\alpha=F / \beta A=2 P_{p} / \beta=9.2 \times 10^{-5} \mathrm{~m} / \mathrm{s}^{2}$, which is equivalent to that of the ion thruster and plasma thruster. 


\subsubsection{Laser Propulsion System}

Nowadays, the space trip business in the private sector aiming at weightless experience is becoming a reality in Europe and the United States. For example, "Space Ship One" or "Space Ship Two" systems are well known at present to the general public (Figure 5).

Now, there are three kinds of space trips such as suborbital trip, orbital trip and round trip around the moon that are being prepared for a space trip, which can be purchased through a travel company or an agency.

- Suborbital trips are aimed at a short-duration weightless experience. That is, the carrier-launch aircraft would take beneath a space vehicle to an altitude of $15 \mathrm{~km}$ and then release it. Shortly after release, the space vehicle would fire its hybrid rocket engine and continue on a ballistic ascent to $110 \mathrm{~km}$ altitude.

- On the other hand, the orbital trip would stay at the International Space Station (ISS) or at a space hotel, carrying out Earth observation and enjoying the weightless experience. The Soyuz spacecraft has stayed at the ISS from the past to the present.

- Round trip around the moon is planned where the Soyuz spacecraft is docked with a moon rocket that is assembled in the ISS. After a stay at the ISS or a space hotel, they will go to the moon with the rocket based on a chemical rocket engine system.

We propose the concept of the Manned Space Transportation Vehicle (MSTV) using a laser thruster in combination with the H-II Rocket for an orbital trip and round trip around the moon. Moreover, using water as propellant is worthy of special mention (Minami \& Uchida, 2013).

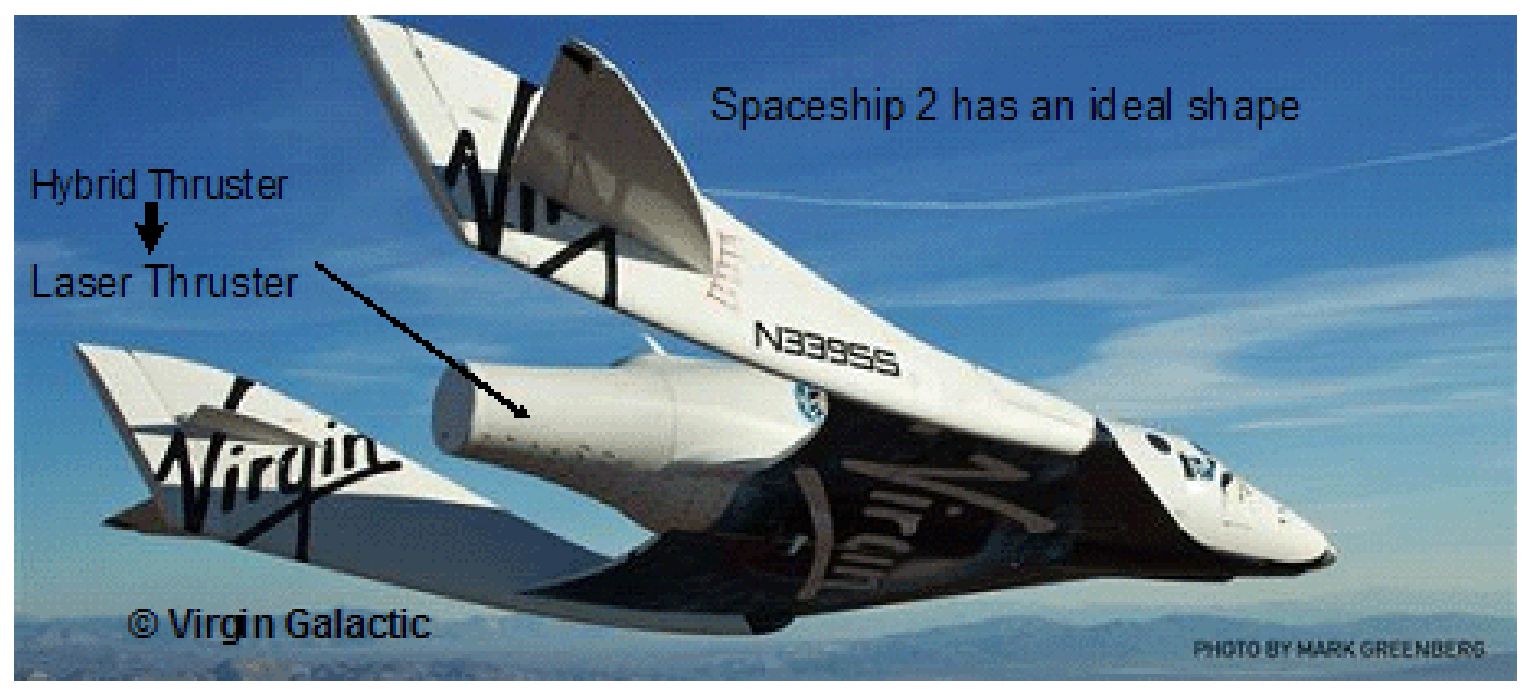

Figure 5. Spaceship 2 with laser thruster 


\section{An example of Laser Thruster (Propellant: vapor)}

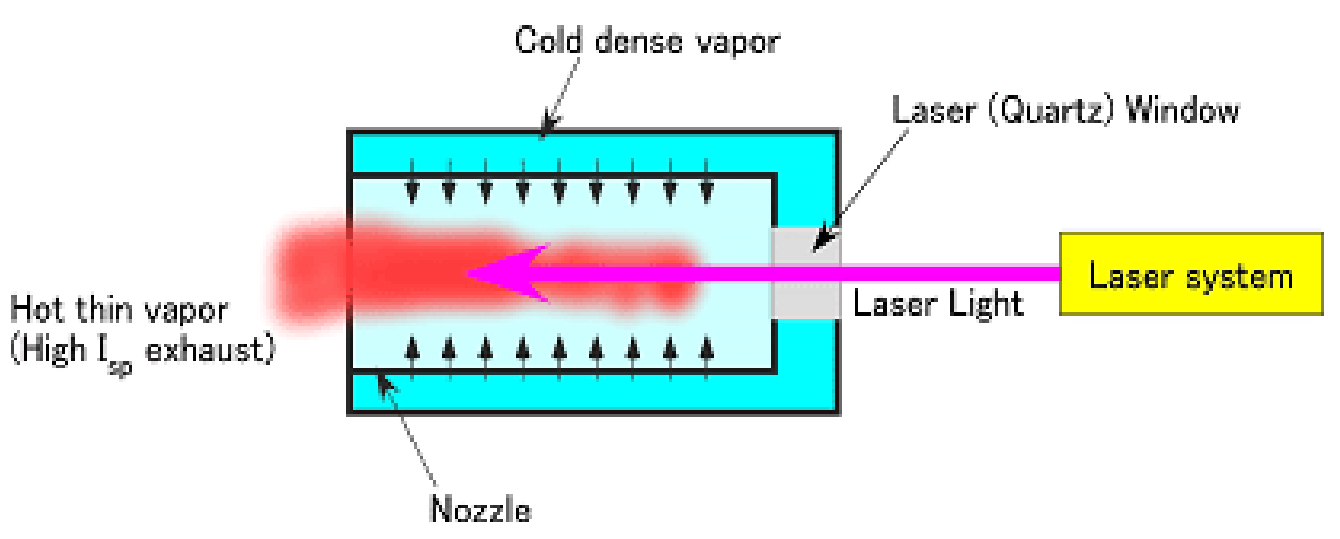

Figure 6. Example of laser thruster

It becomes practical by replacing the spaceship 2 hybrid engine with a laser thruster (Figure 6).

Laser generated thrust and the velocity of ablating gas have been measured in a number of laboratory experiments with a wide range of laser intensities and wavelengths on various target (propellant) materials. It has been clarified that ablation velocities from $100 \mathrm{~m} / \mathrm{s}$ $\left(\mathrm{I}_{\mathrm{SP}}=10 \mathrm{~s}\right)$ to $40 \mathrm{~km} / \mathrm{s}\left(\mathrm{I}_{\mathrm{SP}}=4000 \mathrm{~s}\right)$ are possible by selecting a proper combination of ablating materials and laser conditions, mainly intensity. Laser diode (LD) which has made remarkable technical progress in terms of high power generation is supposed to be a suitable choice for an on-board power source. LD can perform at their best when used in a CW mode and is suitable for generating low $\mathrm{I}_{\mathrm{SP}}$ thruster. On the other hand, a pulsed laser mode generating high peak power is suitable for high $\mathrm{I}_{\mathrm{SP}}$ thruster.

For instance, if the speed of the exhausting gas is increased, large momentum can be generated with a small amount of propellant mass and large amount of laser energy. On the other hand, if the speed is reduced, the same momentum can be obtained by smaller laser energy. The former has a condition of the electric propulsion (speed $10 \mathrm{~km} / \mathrm{s}$ or more of the propulsion gas). In the latter when a large amount of propellant is available and large thrust is needed, where speed of the exhausting gas is $5 \mathrm{~km} / \mathrm{s}$ or less will be adopted. In a laser propulsion system, such engine performance can be switched.

Figure7 depicts scheme of laser engine driven by water propellant using ice/water laser heating. The engine consists of a nozzle, a water tank, the water or ice stored in the nozzle, a laser system and a laser absorber. CW laser or Pulsed laser radiation irradiates water inside the nozzle. Water is supplied through a hole on nozzle from water tank. The size of the hole, in the order of millimeters is adjusted so that water is kept in the tank by its surface tension and repeatedly renewed after laser irradiation. Almost all of laser light is absorbed with a laser absorber and is changed into thermal energy here. The layer of a proper quantity of water has covered the laser absorber then thermal energy blows away the layer of this water and is transformed into kinetic energy. In case that high thrust is required, CW laser radiation 


\section{Macrothink}

irradiates water directly. Since temperature is kept low while a large volume of water is simultaneously heated compared with a pulsed laser, injection of the low-temperature propellant of large mass is made. Therefore, the large momentum coupling coefficient $C_{m}(\mathrm{~N}$. $\mathrm{s} / \mathrm{J}=\mathrm{N} / \mathrm{W}$ ) is realized and a high thrust is obtained. In the case of pulsed laser, due to its high peak power, the surface of water is rapidly heated and injection of high-temperature propellant of small mass is made. Therefore, a high specific impulse ( $\left.\mathrm{I}_{\mathrm{SP}}\right)$ is obtained. In other words, selection between high $\mathrm{C}_{\mathrm{m}}$ system and high $\mathrm{I}_{\mathrm{SP}}$ system can be realized by controlling propellant exhausting velocity. This mechanism corresponds to High $\mathrm{C}_{\mathrm{m}}$ with volume absorbers by $\mathrm{CW}$ laser and High $\mathrm{I}_{\mathrm{SP}}$ with surface absorbers by pulsed laser respectively (Minami \& Uchida, 2009).

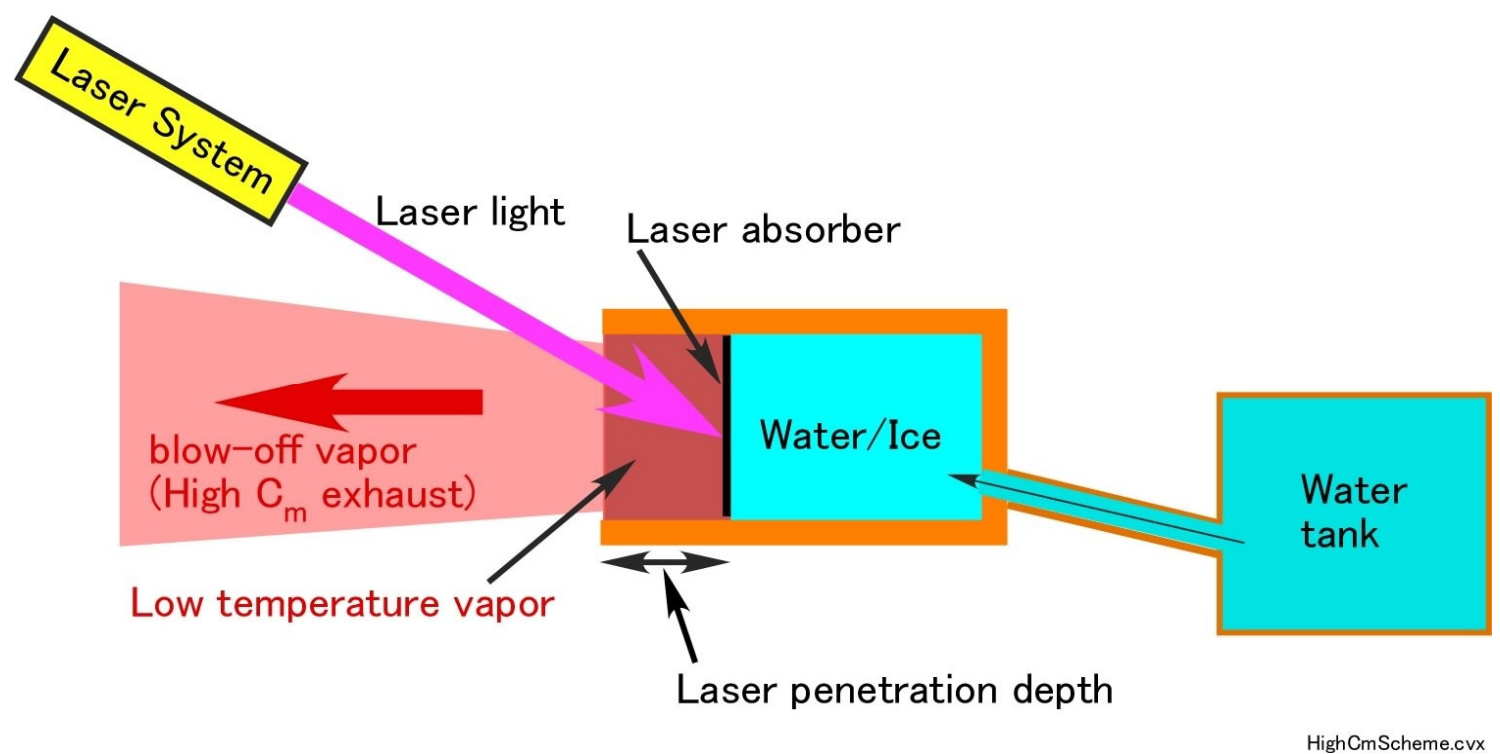

Figure 7. Laser Thruster Using Water

In laser propulsion technology, velocity and fluid conditions of propellant can be controlled by the energy density of laser radiation: by means of the combination of laser parameters such as wavelength, intensity and propellant.

There are two important performance parameters defined for the thrust generation by laser propulsion scheme. They are the specific impulse $\mathrm{I}_{\mathrm{SP}}$ used in the conventional propulsion scheme and momentum coupling coefficient $\mathrm{C}_{\mathrm{m}}(\mathrm{N} \cdot \mathrm{s} / \mathrm{J}=\mathrm{N} / \mathrm{W})$ specific to laser propulsion (Minami \& Uchida, 2015).

$$
\begin{gathered}
C_{m}=\frac{F}{P_{L}}=\frac{\dot{m} V_{E}}{P_{L}}, \\
I_{S P}=\frac{F}{g \dot{m}}=\frac{\dot{m} V_{E}}{g \dot{m}}=\frac{V_{E}}{g},
\end{gathered}
$$

where $C_{m}$ is defined as a ratio of thrust $F$ to incident laser power $P_{L \cdot,} V_{E}$ is exhaust velocity of propellant. 
It is similar to the thrust electric power ratio $(\mathrm{N} / \mathrm{kW})$ of the electric propulsion.

Further, similarly to the electric propulsion, the following relation between thrust $F$ and propulsion efficiency $\eta$, which is defined as the ratio of propellant kinetic energy to the laser energy, is important:

$$
F=\frac{2 \eta P_{L}}{g I_{S P}},
$$

where $\mathrm{g}$ is gravitational acceleration and $P_{L}$ is laser power.

Next, we introduce MSTV (Manned Space Transportation Vehicle) in detail.

By combined use of laser thruster and H-II Rocket, orbital transfer can be performed by the following two steps: (1) MSTV with one crew put into circular orbit in an altitude of $200 \mathrm{~km}$ around the Earth (parking orbit) using H-II Rocket, (2) MSTV is put into circular orbit in an altitude of $400 \mathrm{~km}$ (ISS orbit) from $200 \mathrm{~km}$ circular orbit by using a laser thruster. The H-II Rocket has the following launch capability of payloads for LEO (Low Earth Orbit) at 300km: 10 t (H-II A Rocket), 16.5 t (H-II B Rocket).

Putting a heavy load like an MSTV (5-14 t payload) into the orbit of the ISS or a space hotel directly is technically very difficult.

Figure 8 shows the overview of orbital course to ISS using H-II Rocket and MSTV. First, it is required to put an MSTV into the low earth orbit of an altitude of $200 \mathrm{~km}$. Afterwards, the orbital transfer between the $200 \mathrm{~km}$ circular orbit and the $400 \mathrm{~km}$ circular orbit is performed, and finally MSTV arrives at the ISS or a space hotel at an altitude of $400 \mathrm{~km}$. MSTV uses a laser thruster in which speed control is possible to achieve orbital transfer with high precision accuracy.

When the space tourism by private business is taken into account, Figure 9 shows the outline of the sightseeing tour to the moon via ISS or a Space Hotel. The moon sightseeing at a distance of $384400 \mathrm{~km}$ requires $\Delta \mathrm{V}=3.13 \mathrm{~km} / \mathrm{s}, 1368 \mathrm{~kg}$ of water and a short Moon sightseeing at a distance of $200000 \mathrm{~km}$ requires $\Delta \mathrm{V}=3.05 \mathrm{~km} / \mathrm{s}, 1337 \mathrm{~kg}$ of water. 


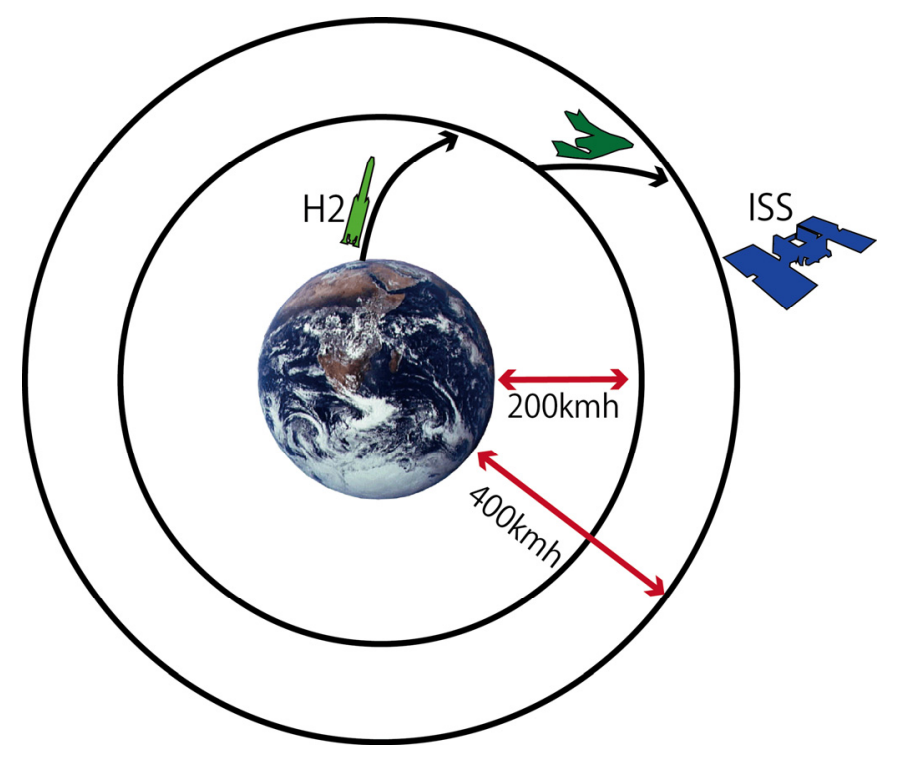

Figure 8. Launch to LEO

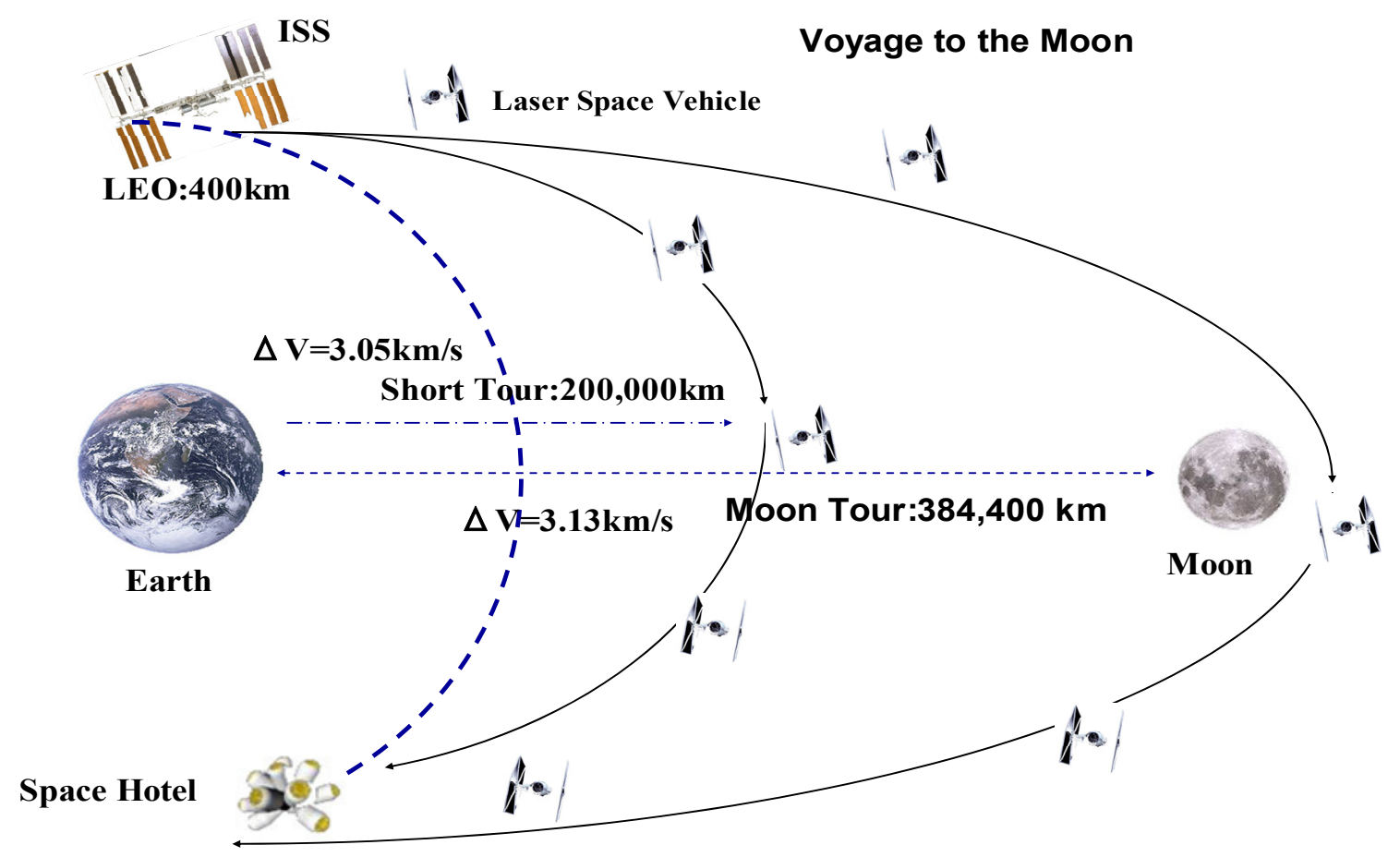

Figure 9. Moon Tour from ISS or Space Hotel

Figure 10 shows the MSTV system block diagram. Thousands of $\mathrm{kW}$ are required for laser power source. The system consists of Laser Diode Stack Array, Laser Collector Optics, Nozzle, Water Tank, Fuel Cell Array for Laser power, Controller and Radiator in the main.

Figure 11 shows the moon sightseeing to fly in the circular orbital altitude of $100 \mathrm{~km}$ from the 


\section{Macrothink Institute ${ }^{\mathrm{TM}}$}

surface of the Moon: Orbital velocity of $1600 \mathrm{~m} / \mathrm{s}$ is necessary.

\section{MSTV System Block(High Thrust Mode)}
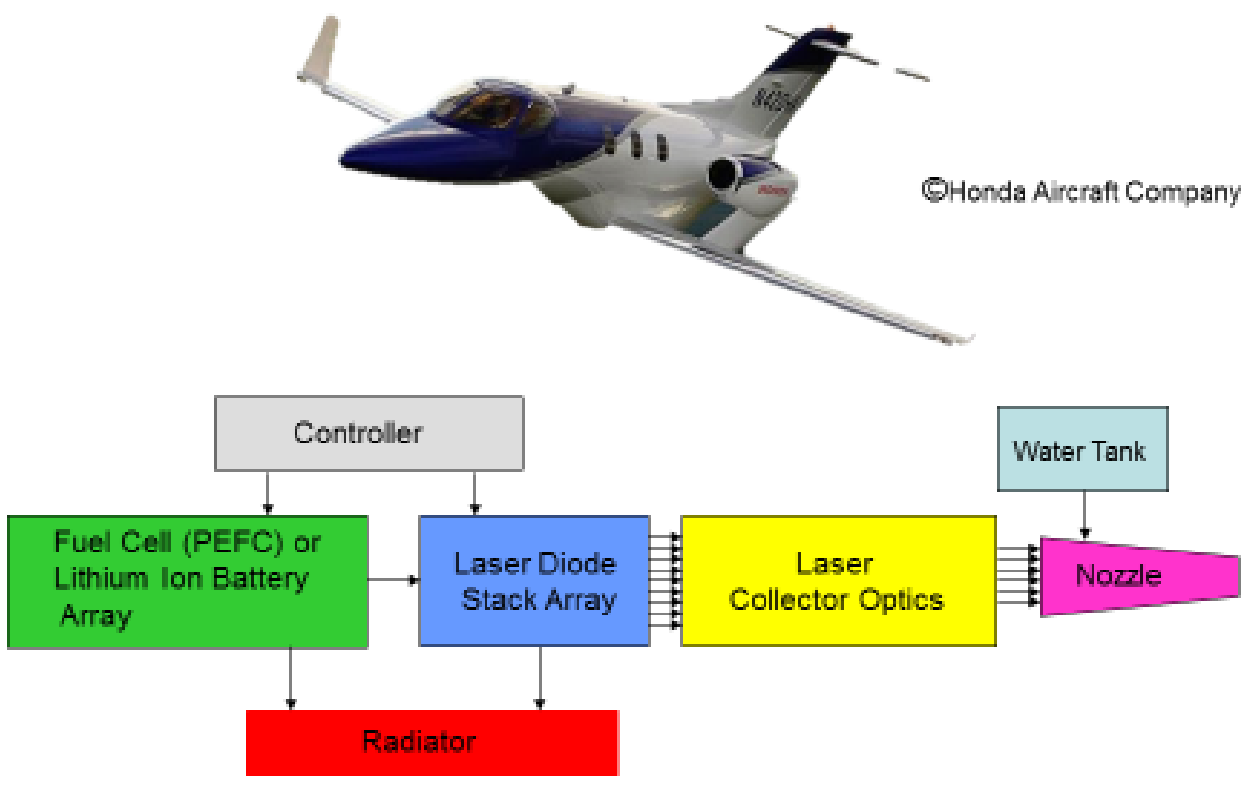

Figure 10. MSTV system block diagram

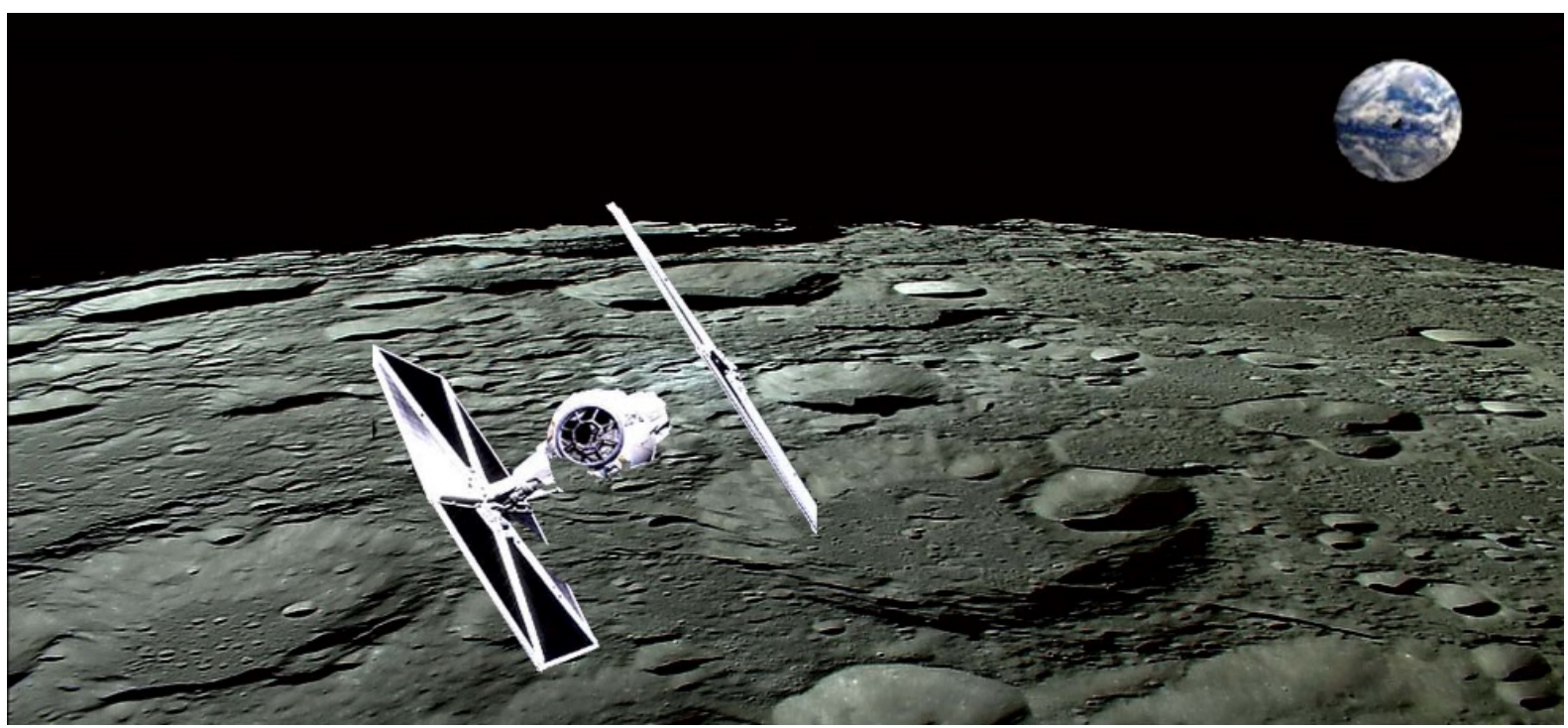

Figure 11. Moon Sightseeing 


\section{Macrothink}

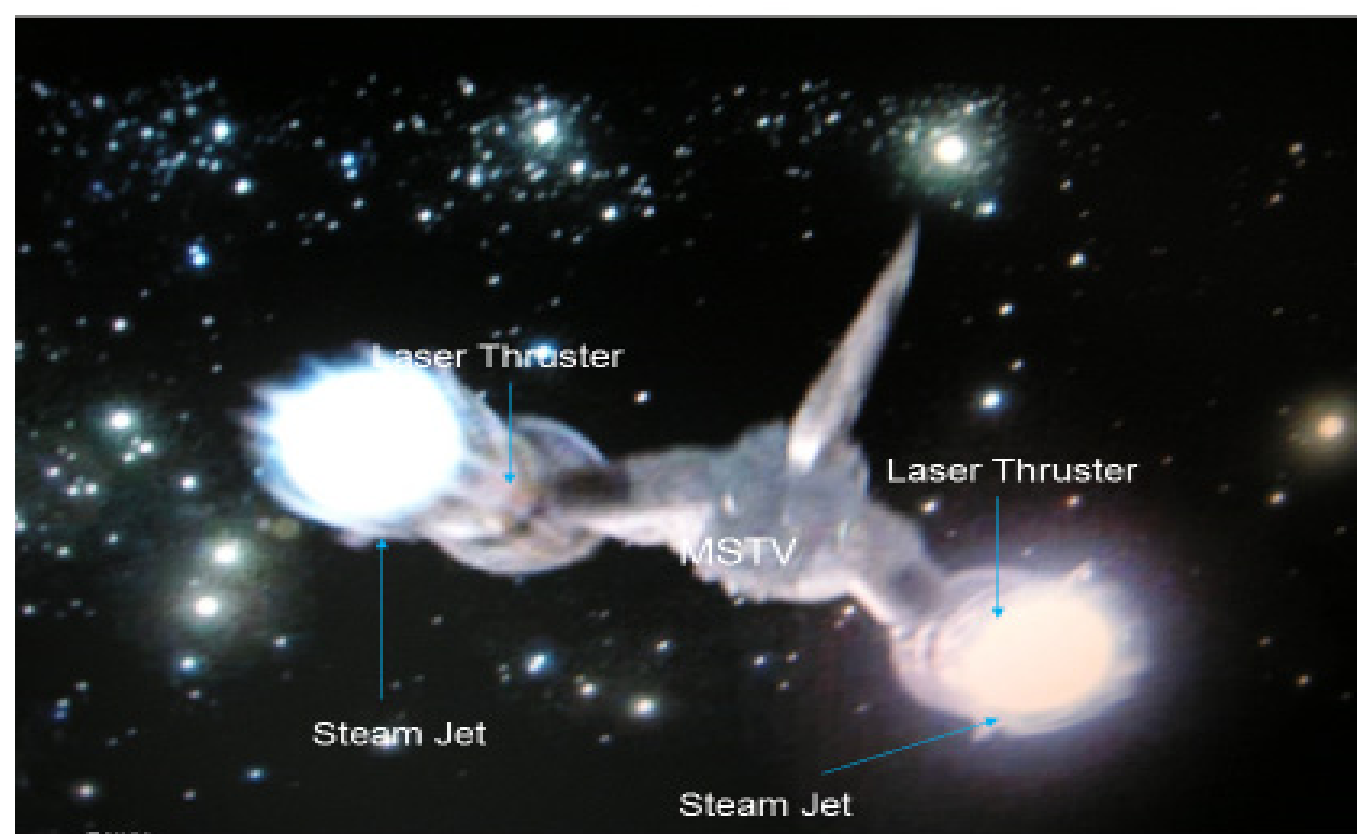

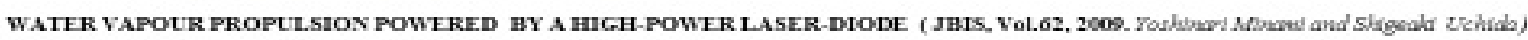

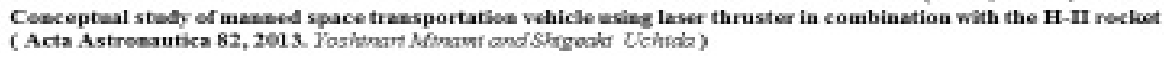

Figure 12. MSTV in space flight

Figure 12 shows an imaginary view of a laser thruster mounted spacecraft flying in space between a planet and its satellites.

\section{Application to Satellite}

\section{- Attitude Control or Trajectory Control}

- Small size laser thruster is used

- Thrust by laser thruster (100W LD) :16mN

$$
212 \mathrm{~W}(1.9 \mathrm{~V}, 112 \mathrm{~A})
$$

- Thrust by ion thruster: $8 \mathrm{mN}$

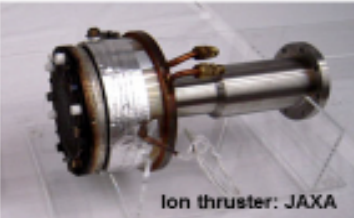

$350 \mathrm{~W}(1.5 \mathrm{kV}, 143 \mathrm{~mA})$

Figure 13. Attitude control laser thruster

Apart from MSTV, small size laser thruster is applied to satellite as attitude control or trajectory control as shown in Figure 13. As compared with MSTV, laser power and power supply becomes small. The required power supply can be used as the same value as ion 
thruster, which is supplied from satellite (Minami, Uchida, \& Takahashi, 2017).

2.1.4 Field Propulsion: Pressure of Field derived from Space-Time

Here, we introduce a new propulsion concept.

The term "Field Propulsion" was firstly notarized by Minami. The detailed concept is summarized in [Y.Minami, "An Introduction to Concepts of Field Propulsion," JBIS, .56, pp.350-359 (2003)] (Minami, 2015).

The field propulsion is defined as follows:

$<$ Definition $>$

Field propulsion is the propulsion method using the field, which is propelled by being pushed or pulled by surrounding space-time as a field. Field propulsion is propelled receiving the propulsive force (i.e., thrust) arises from the interaction of space-time around the spaceship and the spaceship itself, that is, the field propulsion is propelled against space-time structure. The field propulsion principle is based on the assumption that space as a vacuum possesses a substantial physical structure. The field propulsion utilizes the mechanical property of space-time possessing structure (continuum mechanics in the view of the macroscopic structure, statistical mechanics in the view of the microscopic structure). However, a propulsion using the momentum thrust generated by an independent matter, such as plasma, ion, and photon with unrelated to the structure of space-time (or pressure thrust induced by mutual interaction with an independent matter, such as plasma, ion, and photon with unrelated to the structure of space-time) is not applicable to field propulsion. The pressure thrust in field propulsion is different from the pressure thrust in solar sails and light sails induced by receiving the pressure of light.

The most remarkable results attainable through Field Propulsion are as follows: 1) high acceleration such as several ten $G$ can be obtained, 2) theoretical final velocity close to the speed of light, 3) no action of inertial force.

As to item 3), this comes from the thrust as a body force. Since the body force they produce acts uniformly on every atom inside the spaceship, accelerations of any magnitude can be produced with no strain on the crew, i.e., it is equivalent to free-fall. Therefore, the flight patterns such as quick start from the stationary state to all directions in the atmosphere, quick stop, perpendicular turn, and zigzag turn are possible.

Now, a field propulsion system must satisfy the following criteria:

1) conservation of momentum, 2) conservation of energy, 3) ability to induce a unidirectional acceleration of the spaceship, 4) controllability of direction and thrust, 5) sustainability during spaceship motion, 6) effective capability of propelling the spaceship.

\subsection{Physics of Field Propulsion}

In this section, basic principles of field propulsion, the overall concept, and the theory of field propulsion are described. The concept of field propulsion is mainly classified into General Relativistic Field Propulsion and Quantum Field Propulsion (Minami, 2005, 2015, 2017, 2019).

\subsubsection{Propulsion Principle of Field Propulsion}

All existing methods of propulsion systems, i.e., chemical propulsion, electric propulsion (Ion 
thruster, MPD [Magneto Plasma Dynamic] thruster, Hall thruster, ARC jet thruster), laser propulsion, nuclear propulsion are based on expulsion of a mass to induce a reaction thrust. The "momentum thrust" is based on momentum conservation law.

Alternatively, the concept of "Field Propulsion" is propelled by pressure thrust without mass expulsion. The envisaged solar sails and light sails are propelled just by receiving light pressure, but pressure thrust in Field Propulsion refers to a reaction with space-time itself (i.e., the vacuum) to generate a propulsive force. The propulsive force as a pressure thrust arises from the interaction of space-time around the spaceship and the spaceship itself. The spaceship is propelled against space-time structure. The Field Propulsion principle consists in the exploitation of the action of the medium field induced by such interaction and is thus based on some concepts in modern physics to be found in General Relativity, Quantum Field Theory, Quantum Cosmology and Superstring Theory including D-brane to bring about the best propulsive performance.

As shown in Figure 14, the propulsion principle of field propulsion system is not momentum thrust but pressure thrust induced by pressure gradient (or potential gradient) of space-time field (or vacuum field) between bow and stern of spaceship. Since the pressure of vacuum field in the rear vicinity of spaceship is high, spaceship is pushed from vacuum field. Pressure of vacuum field in the front vicinity of spaceship is low, so spaceship is pulled from vacuum field. In the front vicinity of spaceship, the pressure of vacuum field is not necessarily low but the ordinary vacuum field, that is, just only a high pressure of vacuum field in the rear vicinity of spaceship. The spaceship is propelled by this distribution of pressure of vacuum field. Vice versa, it is the same principle that the pressure of vacuum field in the front vicinity of spaceship is just only low and the pressure of vacuum field in the rear vicinity of spaceship is ordinary. In any case, the pressure gradient of vacuum field (potential gradient) is formed over the entire range of spaceship, so that the spaceship is propelled by being pushed from the pressure gradient of vacuum field.

\section{Propulsion Principle}

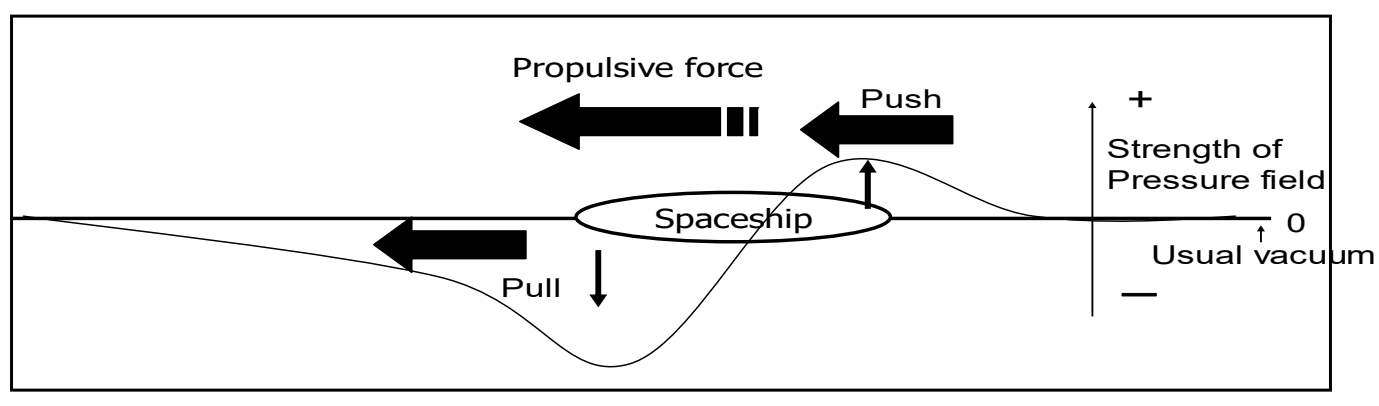

Asymmetrically interaction with the pressure of field creates propulsive force for the spaceship.

The strength of pressure field ahead of the spaceship is diminished and its behind increased, this would result in favorable pressure gradients.

Figure 14. Fundamental propulsion principle of Field propulsion

Here, we must pay attention to the following. Spaceship cannot move unless the spaceship is 
independent of pressure gradient of vacuum field. No interaction is present between pressure gradient of vacuum field and spaceship. Spaceship does not move as long as the propulsion engine generates the pressure gradient or potential gradient in the surrounding area of spaceship, due to the interaction between pressure gradient of vacuum field and spaceship. This is because an action of propulsion engine on space is in equilibrium with a reaction from space. It is consequently necessary to shut off the equilibrium state to actually move the spaceship. As a continuum, the space has a finite strain rate, i.e., speed of light. When the propulsion engine stops generating the pressure gradient of vacuum field, it takes a finite interval of time for the generated pressure gradient of vacuum field to return to ordinary vacuum field. In the meantime, the spaceship is independent of pressure gradient of vacuum field. It is therefore possible for the spaceship to proceed ahead receiving the action from the vacuum field.

In general, a body cannot move carrying, or together with, a field that is generated by its body from the standpoint of kinematics. In other words, the body cannot move unless the body is independent of the field. This is because an action on the field and a reaction from the field are in the state of equilibrium.

As mentioned above, since the propulsion engine must necessarily be shut off for propulsion, the spaceship can get continuous thrust by repeating the alternate ON/OFF change in the engine operation at a high frequency.

Concerning the propulsion principle of field propulsion system, the distribution of field as shown in Figure 14 is fundamental; accordingly, several kinds of propulsion systems have been proposed. Even if any propulsion system is selected, whether the constituents of pressure gradient or potential gradient generated by propulsion engine are curvature, metric, zero-point radiation pressure or entropy, the propulsion principle of field propulsion system is the identical. Furthermore, it should be noted that there is no propulsion theory which exceeds the speed of light.

Further, as is already explained, all propulsion systems based on the momentum thrust receive the reaction thrust by expelling the propellant mass. However, since no propellant is necessary for field propulsion, field propulsion is well called a propellant-less propulsion.

\subsubsection{Basic Physical Concepts applied for Field Propulsion}

The propulsion principle of field propulsion assumes that space as a vacuum possesses a substantial physical structure. Field propulsion is propelled receiving the propulsive force (i.e., thrust) arises from the interaction of the substantial physical structure as space. The substantial physical structure of space is interpreted from both General Relativity in the view of macroscopic structure and Quantum Field Theory in the view of microscopic structure.

Firstly, General Relativity is the geometric theory of gravitation, and the gravitation is explained by curved space. The curvature of space plays an important role. Although the curvature is a number in its mathematic definition, the curvature has relations with continuum mechanics such as expansion, contraction, elongation, torsion and bending. This physical relation indicates that the space as a vacuum can be considered as a kind of elastic body like rubber in continuum mechanics. Therefore, the propulsion system used General Relativity is to be proposed from the standpoint of continuum mechanics. 


\section{Mll Macrothink}

Secondly, space-time as a vacuum is generally viewed as a transparent and ubiquitous infinite empty continuum, upon which physical events take place. However, the quantum field theory and quantum electrodynamics (QED) view the vacuum as possessing vigor and vitality over scales of time and space.

Figure 15 shows the fine structure of space. Such vigor and vitality are the zero-point fluctuations of the vacuum electromagnetic field (vacuum perturbation) as shown in Figure 15 (a), and there exists the continuous creation and annihilation of virtual particle pairs or zero point oscillators (fundamental vacuum of space).

Further, according to the latest quantum optics, although until recently, it has been considered that the control of vacuum perturbation was utterly impossible. At the present day, it is proven that the vacuum perturbation can be controlled by squeezed light technology. Namely, it is possible to increase the energy density locally above the vacuum state and vice versa, decrease the energy density locally below the vacuum state. That is, the squeezed light generates the squeezed vacuum states and yields the coordination geometry of energy density.

Furthermore, strings of superstring theory are considered as the threads of the space-time fabric. Strings seem to be the fundamental element of the substructure or fine structure of space-time. Supposing that the string is the constituent of space-time is suggestive of the existence of possible quantum states for space-time. This indicates that the entropy of space-time can be defined as an assembling of strings. Strings as the constituents of space-time correspond to the polymer chains in the elastic body like Figure 15 (b). Since the statistical entropy is the logarithm of the number of states (i.e., degeneracy of system), it is necessary to consider what kinds of physical state exist.

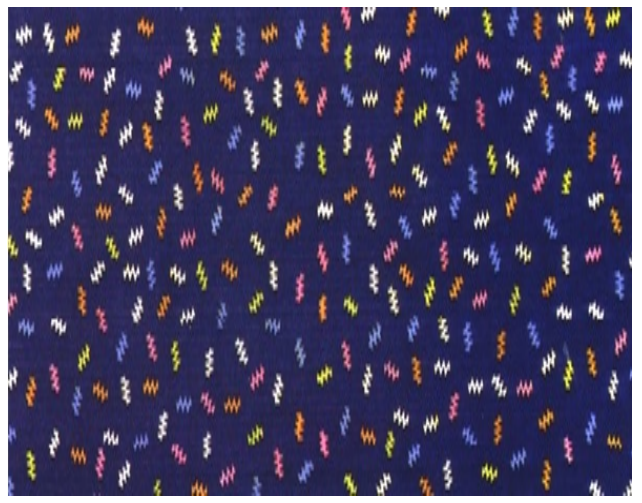

(a)

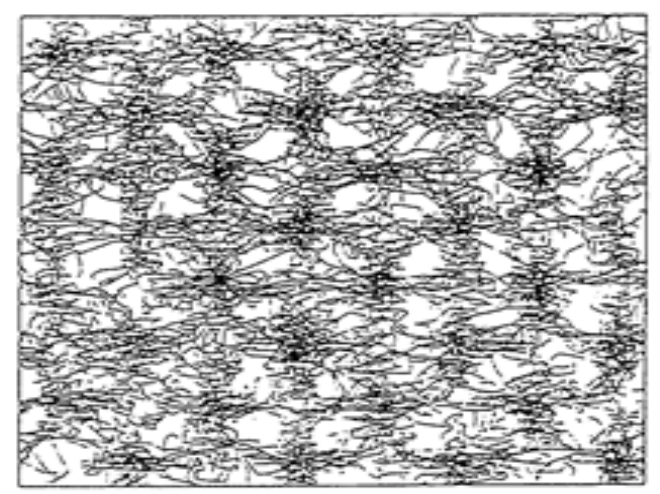

(b)

Small part of zero-point energies of zero point oscillators

Strings as the constituents of space-time

Figure 15. Fine structure of space

Therefore, the propulsion system used Quantum Field Theory is to be proposed from the standpoint of quantum physics.

\subsubsection{ZPF Field Propulsion}

As mentioned previously, the propulsion principle of field propulsion is not momentum thrust 


\section{Macrothink}

but pressure thrust induced by a potential gradient arising from space or vacuum field between the bow and the stern of starship. The propulsive force as a pressure thrust arises from the interaction of space around the starship, which is propelled against the space-time structure. As H. D. Froning shows these figures (Figure 16, Figure 17), the pressure gradient of the vacuum field is formed over the entire range of starship, so that starship is propelled by being pushed from the pressure gradient of the vacuum field (Figure 16).

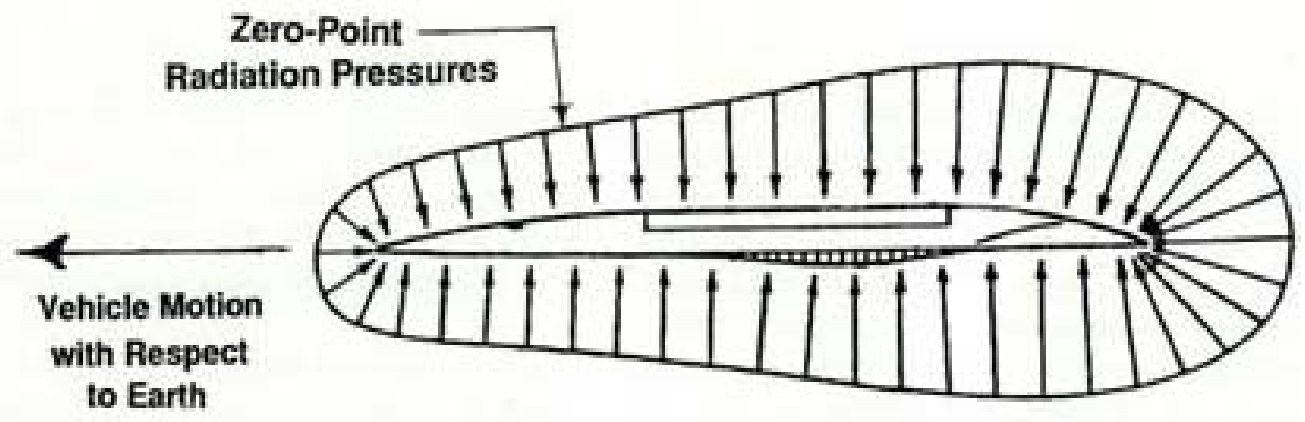

Figure 16. Overview of ZPF field propulsion

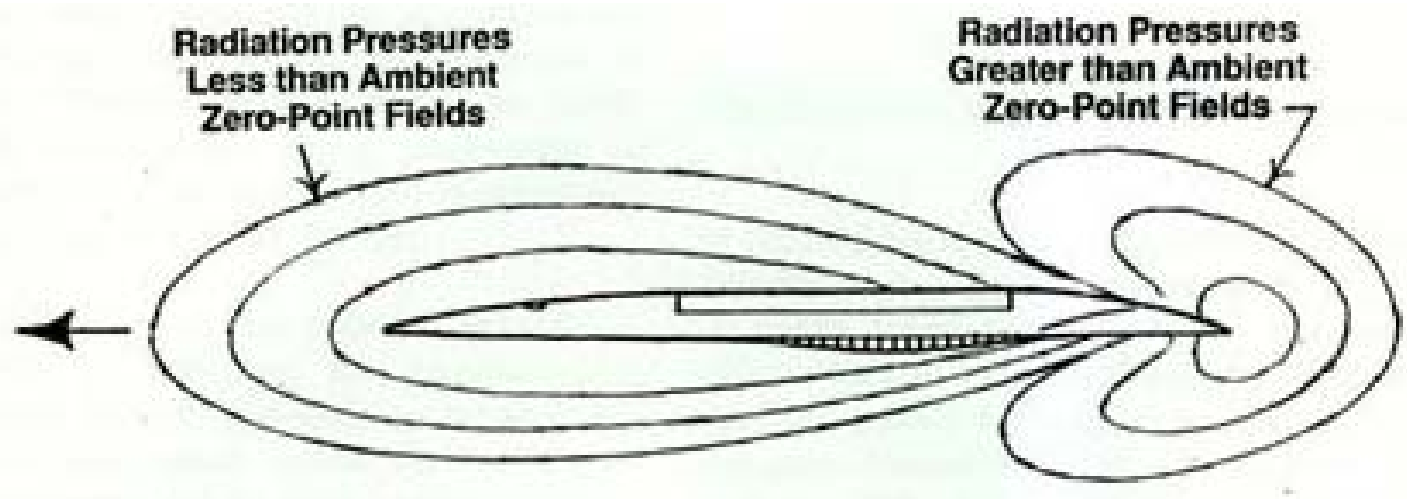

Figure 17. ZPF field propulsion principle

Pressure gradient is generated by gradient of vacuum energy density. The zero-point pressures (vacuum energy density) are less than ambient zero-point fields in the bow of starship, on the other hand, zero-point pressures (vacuum energy density) are greater than ambient zero-point fields in the stern of starship. Therefore, the starship is propelled by being pushed from the zero-point pressure of the vacuum field in the stern of starship (Figure 17).

The following Figure 18 shows the Evolution of field propulsion. 


\section{MInstitute ${ }_{\text {Imk }}^{\text {Macrothink }}$}

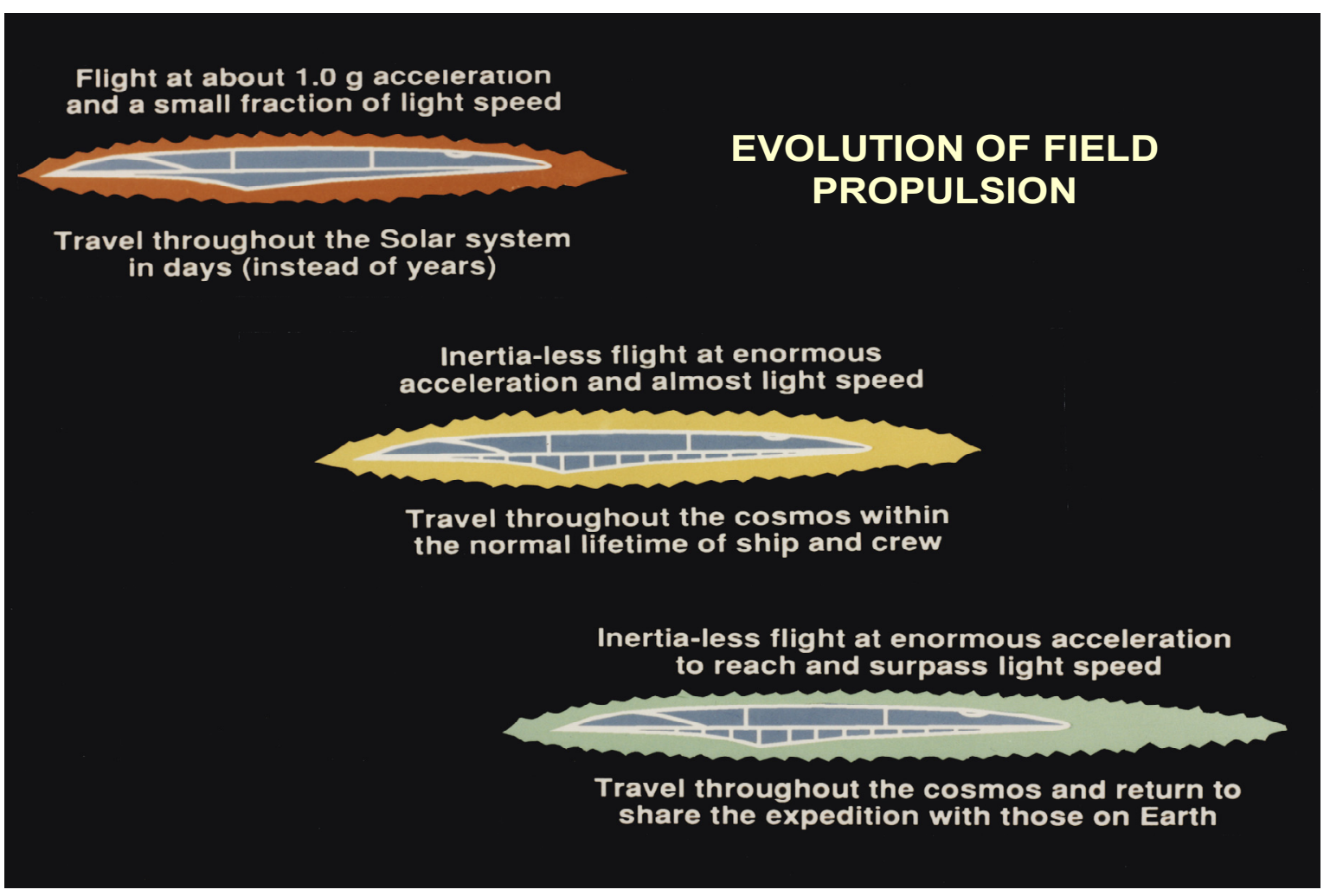

Figure 18. Evolution of field propulsion: from slower solar system flight to swift cosmic exploration (H.D.Froning)

The description of "to reach and surpass light speed" described at the bottom of the Figure 18 means that the light speed is apparently exceeded by combination with the navigation theory shown in Chapter 3: It does not mean that the speed of light is actually exceeded.

\subsubsection{Space Drive Field Propulsion}

As a representative example of a well-studied field propulsion system, space drive propulsion theory induced by space curvature based on the supposition that space is an infinite continuum like the elastic body is introduced (Minami, 1997, 1998, 2014).

Space drive propulsion system is one of field propulsion system utilizing the action of the medium of strained or deformed field of space. The curvature of space plays a significant role for the propulsion theory.

Assuming that a space vacuum is an infinite continuum, the propulsion principle utilizes the pressure field derived from the geometrical structure of space, by applying both continuum mechanics and General Relativity to space. The propulsive force is a pressure thrust that arises from the interaction of space-time around the spaceship and the spaceship itself; the spaceship is propelled against the space-time continuum structure. This means that space can be considered as a kind of transparent elastic field. That is, space as a vacuum performs the motions of deformation such as expansion, contraction, elongation, torsion, and bending. The latest expanding universe theory (Friedmann, de Sitter, inflationary cosmological model) supports this assumption. Space can be regarded as an elastic body like rubber. 
General Relativity implies that space is curved by the existence of energy (mass etc.) and based on Riemannian geometry. If we admit this space curvature, space is assumed as an elastic body. According to continuum mechanics, the elastic body has the property of the motion of deformation such as expansion, contraction, elongation, torsion, and bending. General Relativity uses just only the curvature of space. Expansion and contraction of space are used in cosmology.

The principle of space drive propulsion system is derived from General Relativity and the theory of continuum mechanics. We assume the so-called "vacuum" of space as an infinite elastic body like rubber. The curvature of space plays a significant role in propulsion theory. From the gravitational field equation, the strong magnetic field, as well as, mass density generates the curvature of space, and this curved space region produces the uni-directional acceleration field. The spaceship in the curved space can be propelled in a single direction. Since the force they produce acts uniformly on every atom inside the spaceship, accelerations of any magnitude can be produced with no strain on the crews, that is, there is no action of inertial force because the thrust is a body force (i.e., it is equivalent to free-fall). Minami derived the equation of curvature of space induced by magnetic field in 1988. It was found that this equation was in accordance with the equation that Levi-Civita considered (i.e., the static magnetic field creates scalar curvature) by Minami in 1995.

Since the propulsion mechanism used magnetic field, in the beginning, is easy to understand, we explain it using the magnetic field. At present, space drive propulsion does not need the strong magnetic field under the favor of de Sitter solution.

The principle of the space drive propulsion system is summarized in the following.

First of all, it is necessary for the space to be curved. Because the curvature $R^{00}$ of flat space is zero (strictly speaking, only 20 independent components of Riemann curvature tensor $R_{p i j k}$ are zero), then the acceleration $\alpha$ becomes zero. Such a curved space is generated not only by mass density but also by magnetic field or electric field. In case that the intensities of the magnetic field $\mathrm{B}$ and the electric field $\mathrm{E}$ are equal, the value of $\left(1 / 2 \cdot \varepsilon_{0} E^{2}\right)$ is about seventeen orders of magnitude smaller than the value of $\left(B^{2} / 2 \mu_{0}\right)$. As a consequence, the electric field only negligibly contributes to the spatial curvature as compared with the magnetic field. Accordingly, it is effective that space can be curved by magnetic field. Since the region of curved space produces the field of acceleration, the massive body existing in this acceleration field (i.e., curved space region), is moved by Newton's second law.

In the latest cosmology, the terms vacuum energy and cosmological term " $\Lambda g^{i j}$ " are used synonymously. $\Lambda$ is known as the cosmological constant. The term with the cosmological constant is identical to the stress-energy tensor associated with the vacuum energy. The properties of vacuum energy, i.e., cosmological term are crucial to the expansion of the Universe, that is, to inflationary cosmology.

In the beginning, the acceleration generated by the curvature of space derived from strong 
magnetic field based on external and internal Schwarzschild solution was studied. However, superior acceleration based on de Sitter solution is obtained and presented at $47^{\text {th }}$ IAF in 1996. The details are published in JBIS, Vol.50 (Minami, 1997) and presented at STAIF-98 in 1998 (Minami, 1998).

At the present day, the space drive propulsion system is based on the de Sitter solution as follows:

The acceleration derived from the de Sitter solution does not require a strong magnetic field. At the present day, space drive propulsion system based on the de Sitter solution needs not a strong magnetic field but the technology to excite space.

Additionally, the inflationary universe which shows the rapid expansion of space is based on the phase transition of vacuum exhibited by the Weinberg-Salam model of the electroweak interaction. The vacuum has the property of a phase transition, just like water may become ice and vice versa. This shows that a vacuum possesses a substantial physical structure such as matter. It coincides with the precondition of a space drive propulsion principle.

As is well known in cosmology, the expansion rule of the universe is governed by the Friedman's equations and the Robertson-Walker metric. The propulsion principle for this space drive is newly studied from another angle, that is, the pressure of the field induced by rapid local expansion of space is completely considered in the propulsion principle based on the latest cosmology.

Acceleration of curved space and curvature of space is obtained:

$$
F^{3}=F=m \alpha=m \sqrt{-g_{00}} c^{2} \int_{a}^{b} R^{00}(r) d r=m \sqrt{-g_{00}} c^{2} \Gamma_{00}^{3}
$$

where $\alpha$ : acceleration $\left(\mathrm{m} / \mathrm{s}^{2}\right), g_{00}$ : time component of metric tensor, a-b: range of curved

space region(m), $r$ : direction of the radius of curvature, $c$ : velocity of light, $R^{00}$ : major component of spatial curvature $\left(1 / \mathrm{m}^{2}\right)$.

Equation (10) indicates that the acceleration field $\alpha$ is produced in curved space. The intensity of acceleration produced in curved space is proportional to the product of spatial curvature $R^{00}$ and the length of curved region (point "a" to point " $\mathrm{b}$ ").

The acceleration of space drive propulsion system is based on the solutions of de Sitter cosmological solution of gravitational field equation given by:

$$
\alpha=\frac{2 \pi G \lambda}{3 c^{2}} \phi_{0}^{4}=1.6 \times 10^{-27} \lambda \phi_{0}^{4} .
$$

where $\alpha$ is acceleration $\left(\mathrm{m} / \mathrm{s}^{2}\right), G$ is the gravitational constant, $c$ is the velocity of light, $\phi_{0}$ is non- zero vacuum expectation value of field, $\lambda$ is arbitrary Higgs self-coupling in the Higgs potential ( $\lambda$ is not known and is not determined by a gauge principle, presumably $\lambda \geq 1 / 10$ ).

For these equations derivation, please see reference.

A condensed summary of the propulsion principle of space drive propulsion system is shown as Figure 19. 


\section{SPACE DRIVE PROPULSION SYSTEM}

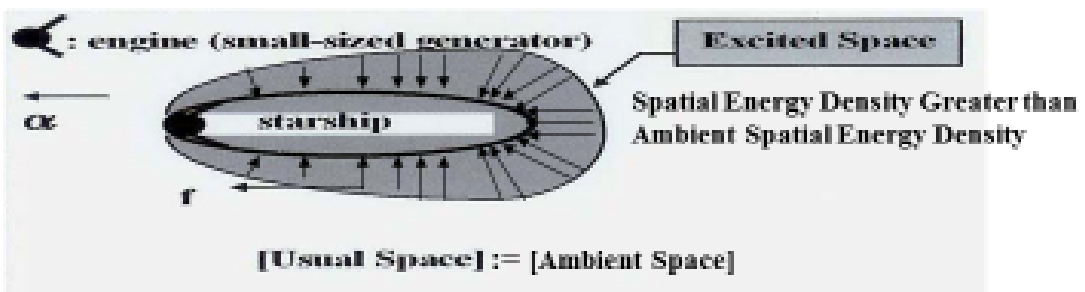

- Curvature of SPACE $\left(\mathrm{R}^{00}\right)$ plays a significant role for propulsion theory (Y.Minami:1988).

$$
\begin{gathered}
F^{i}=m \sqrt{-g_{00}} c^{2} \Gamma_{00}^{i}=m \alpha^{i}=m \sqrt{-g_{00}} c^{2} \int_{a}^{b} R^{00}\left(x^{i}\right) d x^{i} \\
R^{00}=\frac{4 \pi G}{\mu_{0} c^{4}} \cdot B^{2} \quad \begin{array}{l}
\text { Both strength of curvature and } \\
\text { its extent (volume) are important. }
\end{array}
\end{gathered}
$$

- Acceleration induced by de Sitter solution is found in 1996 by Minami : constant acceleration $\alpha$ (i.e. no tidal force inside of the starship).

$$
\begin{gathered}
\alpha=\frac{2 \pi G \lambda}{3 c^{2}} \phi_{0}{ }^{4}=1.6 \times 10^{-27} \lambda \phi_{0}{ }^{4} \\
\Phi_{0} \text { : non-zero vacuum expectation value of field }
\end{gathered}
$$

Figure 19. A condensed summary of space drive propulsion principle

\subsubsection{Mars Exploration by Field Propulsion}

Prospects of Mars Exploration is shown as Figure 20.

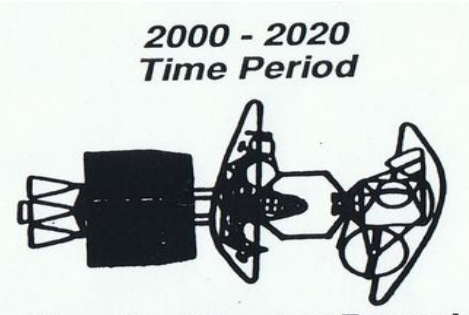

Chemical Rocket Propulsion Expendable Multi-Stage Vehicle Transit Time 250 Days

\section{TYPICAL MARS TRANSPORTATION POSSIBILITIES}

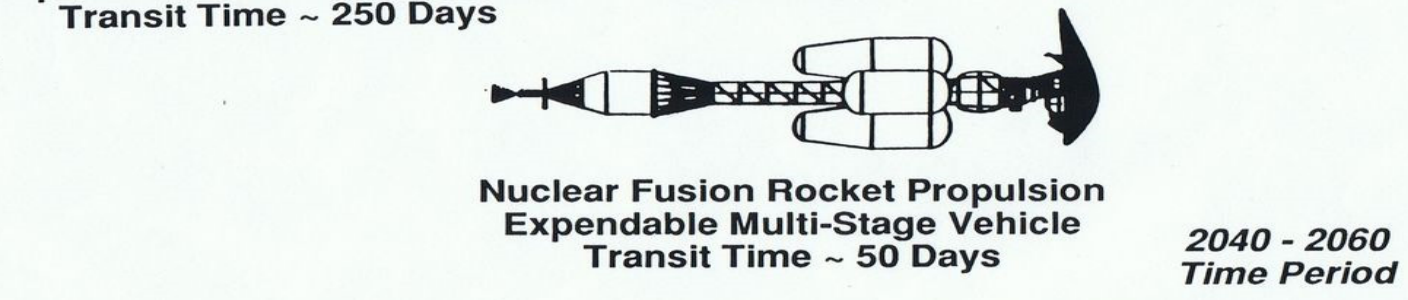

* Travel Distance $=0.52 \mathrm{AU}$ Thrusting During Entire Flight

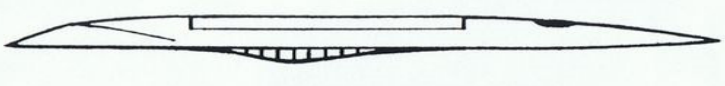

Field Propulsion Single Reusable Stage Transit Time 5 Days*

Figure 20. MARS Transportation Possibilities (H.D.Froning) 


\section{Macrothink}

\section{STAR FLIGHT Method: To the Stars}

\subsection{Three Ways to the Interstellar Travel}

Three methods are considered to reach the star rapidly. The basic principle is the following equation which is known to every one:

$$
L_{\text {star }}=V_{\text {starship }} \times t
$$

where $\mathrm{L}_{\text {star }}$ is the distance to star, $\mathrm{V}_{\text {starship }}$ is the speed of starship, $\mathrm{t}$ is the time.

The distance to a stellar system " $\mathrm{L}_{\text {star }}$ " is enormous. An extremely long time is required, even if the starship would travel at the speed of light "c".

To reach the star rapidly, three parameters, such as "speed", "distance" and "time" shall be controlled.

$$
1) .<\text { Change speed }>\quad L_{\text {star }}=(n c) \times t
$$

where "nc" is n-fold increase in speed of light "c". Here, $\mathrm{n}$ is real number greater than 1.

There is no propulsion theory exceeds the speed of light, moreover, Special Relativity restricts the maximum speed to the speed of light; therefore this method is impossible.

\section{2). <Change distance>}

$$
\frac{L_{\text {star }}}{n}=c \times t
$$

The so-called "wormhole" is utilized (Forward, 1989). By using wormhole, shorten the distance as $L_{\text {star }} / n \approx \mathrm{a}$ few meters, as shown in Figure 21 . For example, one meter in a wormhole corresponds to a few light years in actual space.

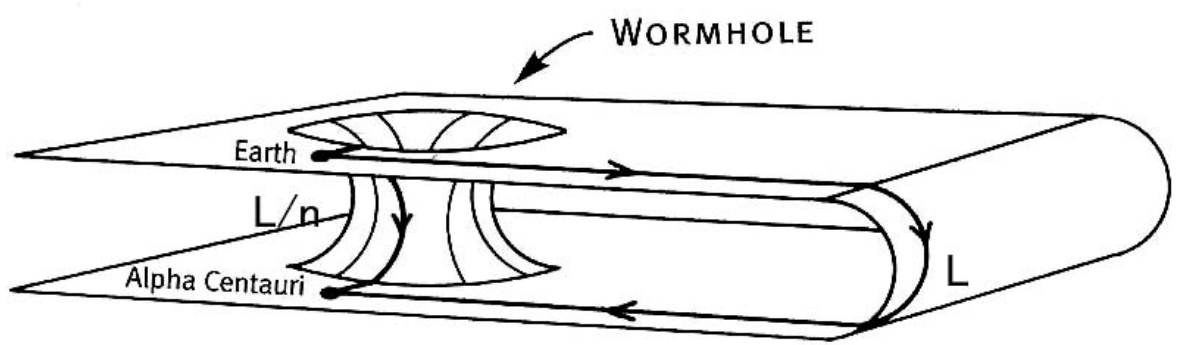

Figure 21. A wormhole creates a shortcut from Earth to Alpha Centauri

$$
\text { 3). <Change time }>\quad L_{\text {star }}=c \times(n t)
$$

The time " $t$ " in an imaginary time hole is equivalent time of $n$-fold time in actual space, as shown in Figure 22.

For example, one second in an imaginary time hole corresponds to one million seconds in actual space. 


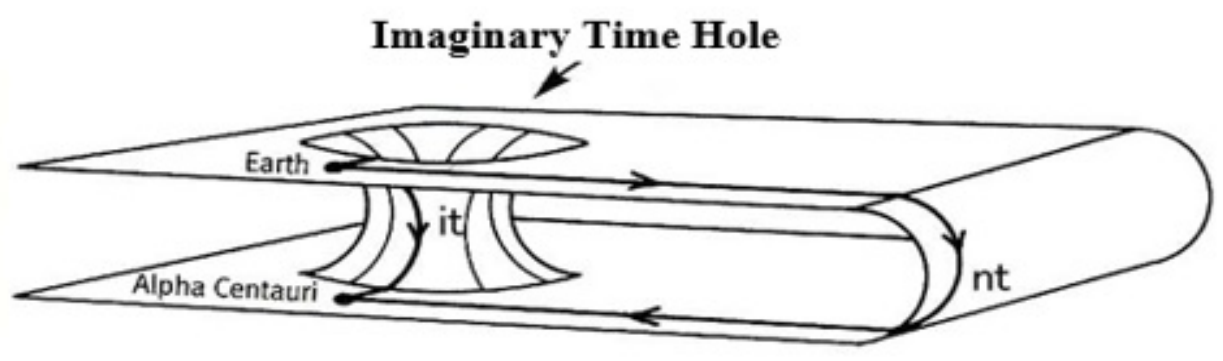

Figure 22. An Imaginary Time Hole creates a shortcut from Earth to Alpha Centauri

Subsequently, interstellar travel through the imaginary time hole is described as the following section.

\subsection{Hyper-Space Navigation (Time Hole Navigation)}

Figure 23 shows the plane of existence of ordinary $x$-ct space-time. This is with a vertical coordinate $\mathrm{ik} \tau$, which is orthogonal to those which describe space travel (x) and time travel (ct) on an x-ct space-time plane of existence. And this x-ct plane is seen to be embedded in the volume of the higher-dimensional x-ct-ik $\tau$ realm rising above it (Froning, 1983).

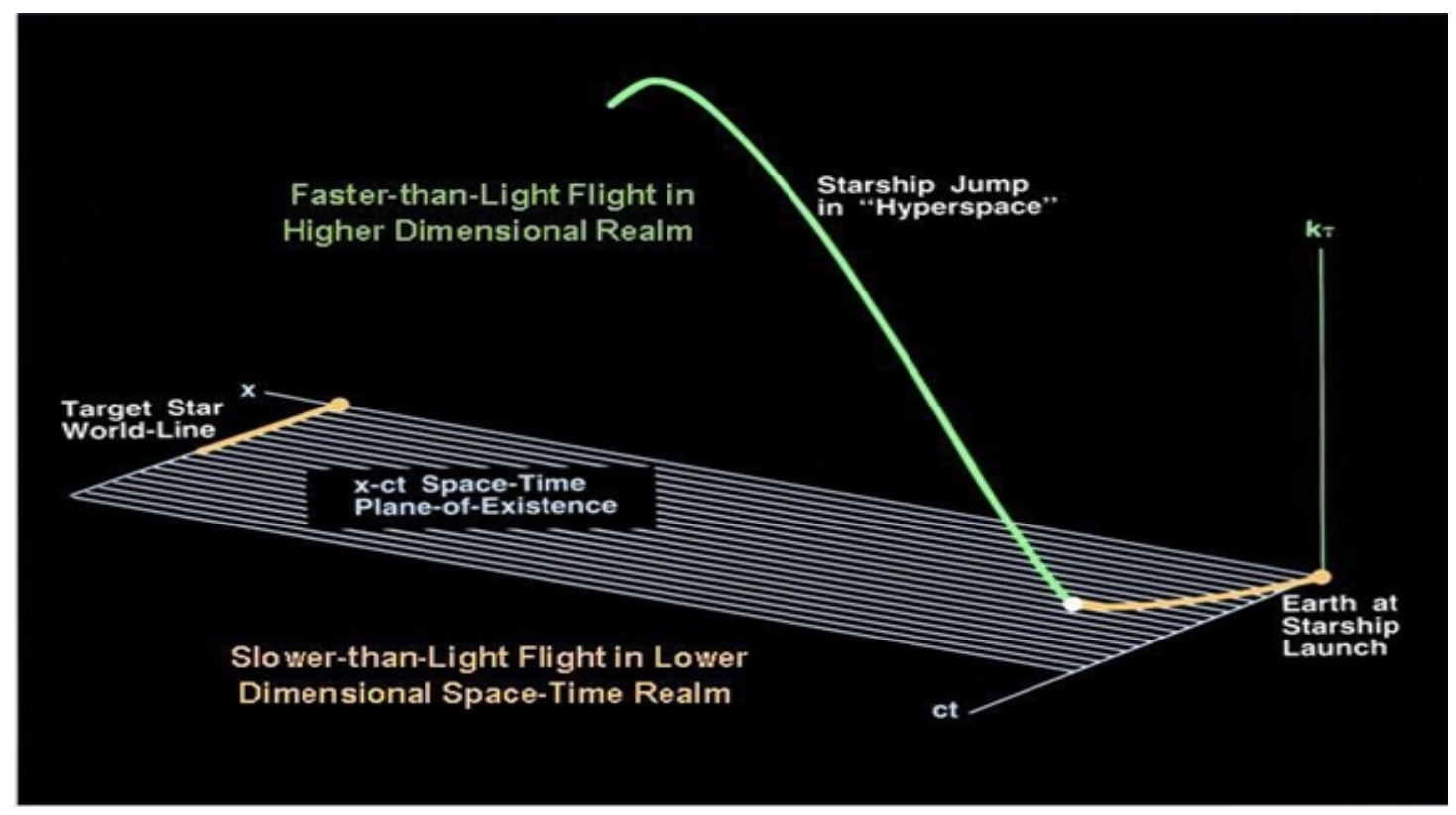

Figure 23. Trajectory faster-than-light flight (H.D.Froning)

In general, the property of space is characterized by a metric tensor that defines the distance between two points. Here, space is divided into two types. Actual physical space that we live in is a Minkowski space, and the world is limited by Special Relativity. It is defined as "Real-Space". Here as a hypothesis, an invariant distance for the time component of Minkowski metric reversal is demanded. This is not a mere time reversal. It is defined as "Hyper-Space". The invariance is identical with the symmetries. Symmetries in nature play many important roles in physics. From this hypothesis, the following arises: the properties of 
the imaginary time $\left(\mathrm{x}^{0}=\mathrm{ict} ; \mathrm{i}^{2}=-1\right)$ are required as a necessary result in Hyper-Space. Here, " $\mathrm{i}$ " denotes the imaginary unit and "c" denotes the speed of light. The time " $t$ " in Real-Space is changed to imaginary time "it" in Hyper-Space. However, the components of space coordinates $(\mathrm{x}, \mathrm{y}, \mathrm{z})$ are the same real numbers as the Real-Space. From the above, it is seen that the real time $\left(x^{0}=c t\right)$ in Real-Space corresponds to the imaginary time $\left(x^{0}=i c t\right)$ in Hyper-Space. The imaginary time direction is at right angles to real time. This arises from the symmetry principle on the time component of Minkowski metric reversal (Minami, 1993, 2003, 2014).

\subsection{Star Flight for Stellar System}

Next, a comparison is made between interstellar travel by Special Relativity and Hyper-Space Navigation. The condition is the same for both cases of navigation, that is, the distance between the earth and the star is 410 light years (i.e., Pleiades star cluster) and the velocity of starship is 0.99999 c.

[Special Relativity allows the following (see Figure 24)]:

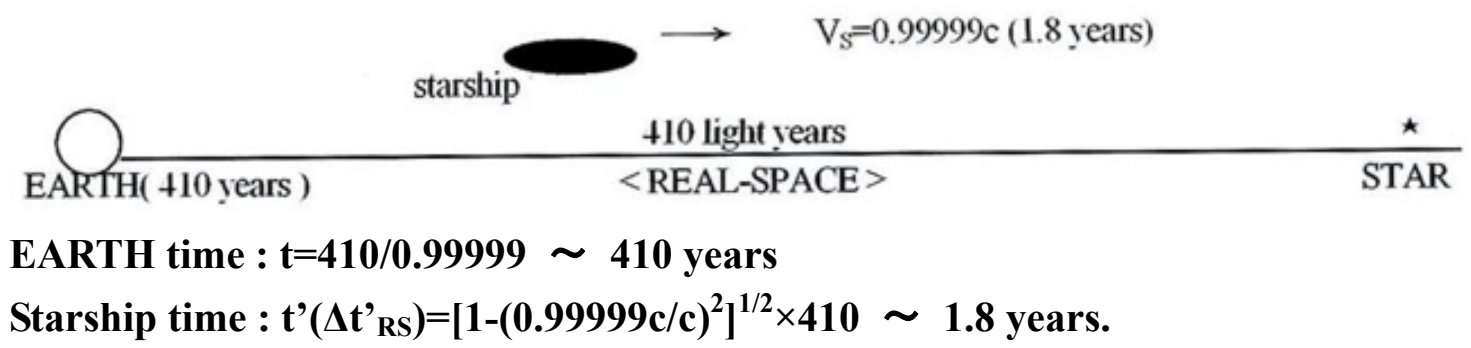

Figure 24. Interstellar Travel by Special Relativity

A starship can travel to star 410 light years distant from us in 1.8 years. However, there exists a large problem as is well known, i.e., the twin or time paradox. If the starship travels at a velocity of $0.99999 \mathrm{c}$, it will arrive at the Pleiades star cluster 1.8 years later. It will seem to the crews in the starship that only 1.8 years have elapsed. But to the people on the earth, it will have been 410 years. Namely, since the time gap between starship time and earth time is so large, the crew coming back to the earth will find the earth in a different period. This phenomenon is true in our Real-Space. Interstellar travel by this method is non-realistic, i.e., it would just be a one-way trip to the stars.

[Hyper-Space Navigation allows the following (see Figure 25)]:

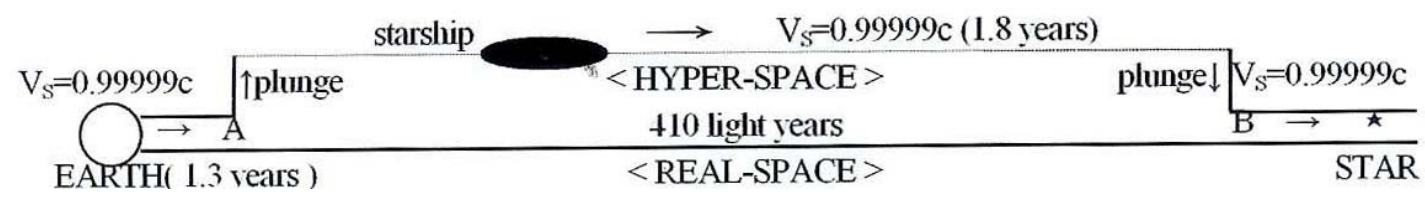

Starship time: $t^{\prime}\left(\Delta t^{\prime}{ }_{H S}\right)=1.8$ years

EARTH time: $t\left(\triangle t_{\mathrm{EHS}}\right)=\left(1 /\left[1+(0.99999 \mathrm{c} / \mathrm{c})^{2}\right]^{1 / 2}\right) \times 1.8=(1 / \sqrt{ } 2) \times 1.8 \sim 1.3$ years

Range: $L=0.99999 \mathrm{c} \times 1.3 \times\left(\left[1+(0.99999 \mathrm{c} / \mathrm{c})^{2}\right]^{1 / 2} /\left[1-(0.99999 \mathrm{c} / \mathrm{c})^{2}\right]^{1 / 2}\right)=0.99999 \mathrm{c} \times 1.3 \times 316 \sim 410$ light years.

Figure 25. Interstellar Travel by Hyper-Space Navigation 
A starship can travel to the stars 410 light years distant in 1.8 years: Due to the continuity of starship time between Real-Space and Hyper-Space.

During Hyper-Space navigation of 1.8 years, just 1.3 years have passed on the earth. Therefore, the time gap between starship time and earth time is suppressed. After all, the range and travel time of starship is the same for both kinds of navigation, and travel to the stars 410 light years away can occur in just 1.8 years in both cases. However, by plunging into Hyper-Space featuring an imaginary time, i.e., Euclidean space property, just 1.3 years, not 410 years, have passed on the earth. There is no time gap and no twin or time paradox such as in Special Relativity. Additionally, a starship can travel to the star Sirius 8.7 light years distant from us in 0.039 years (14 days). During Hyper-Space navigation of 14 days, just 0.028 years ( 9 days) have passed on the earth.

Figure 26 shows such a realistic method for the interstellar travel using Hyper-Space navigation system (i.e., Time Hole; Figure 22). In order to reach the target star, the starship which left the Earth at a velocity of approximately $0.1 \mathrm{c}$ to $0.2 \mathrm{c}$ moves and escapes completely from the Solar System (with Figure 26). After that, the starship is accelerated to nearly the speed of light in Real-Space and plunges into Hyper-Space at point A. In Hyper-Space, the time direction is changed to the imaginary time direction, and the imaginary time direction is at right angles to real time. The course of starship is in the same direction, i.e., $\mathrm{x}$-axis.

With the help of derived equations (these equations are omitted in this paper: refer to (Minami, 1993, 2003, 2014)), the crew can calculate the range by the measurement of starship time. After the calculated time has just elapsed, the starship returns back to Real-Space from Hyper-Space at a point B nearby the stars. Afterward, the starship is decelerated in Real-Space and reaches the target star. It is immediately seen that the causality principle holds. Indeed, the starship arrives at the destination ahead of ordinary navigation by passing through the tunnel of Hyper-Space (Time Hole). The ratio of tunnel passing time to earth time is 1.4:1 and both times elapse. Hyper-Space navigation method can be used at all times and everywhere in Real-Space without any restrictions to the navigation course.

Concerning a concept on technical method of plunging into Hyper-Space and returning back to Real-Space, the following study is necessary: 1) Many-Particle Systems for Starship, 2) Wave function of Starship by Path Integrals, 3) Quantum Tunneling Effect, 4) Reduction of Wave function, 5) Starship Information Content Restoring.

While the conceptual framework discussed above is highly speculative, it is in the wake of most of the current international trends on the subject of "Interstellar Travel". As a matter of fact, the problem of interstellar travel consists much more in navigation theory than in propulsion theory.

Because, there is no propulsion theory capable of causing a starship to travel at a velocity faster than the speed of light. 


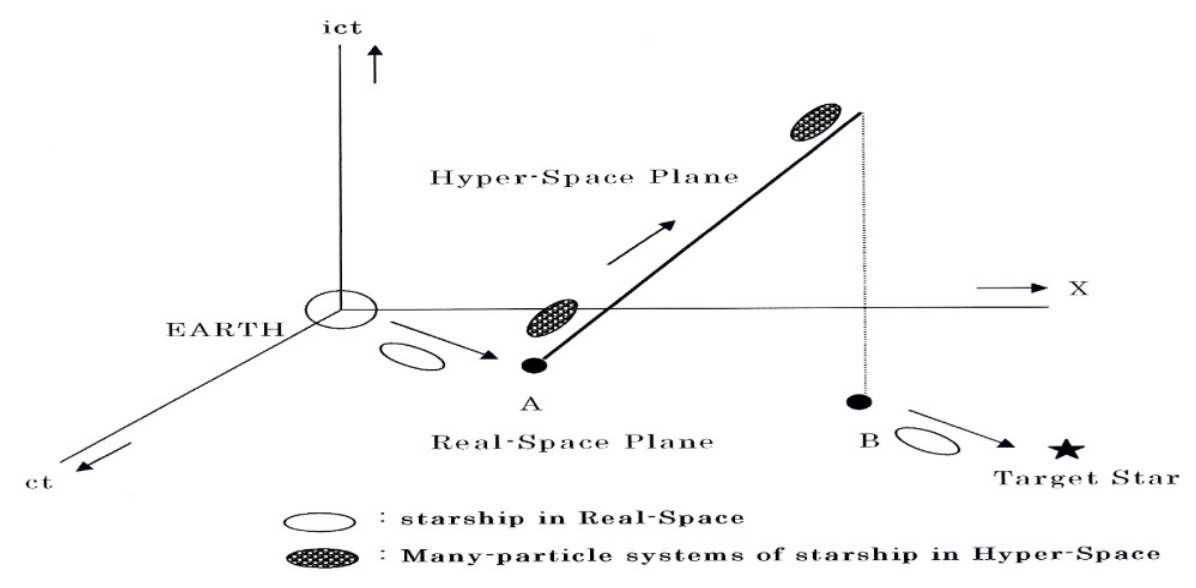

Figure 26. Interstellar travel to the star

Also, Figure 27 schematically shows the navigation of Figure 26.

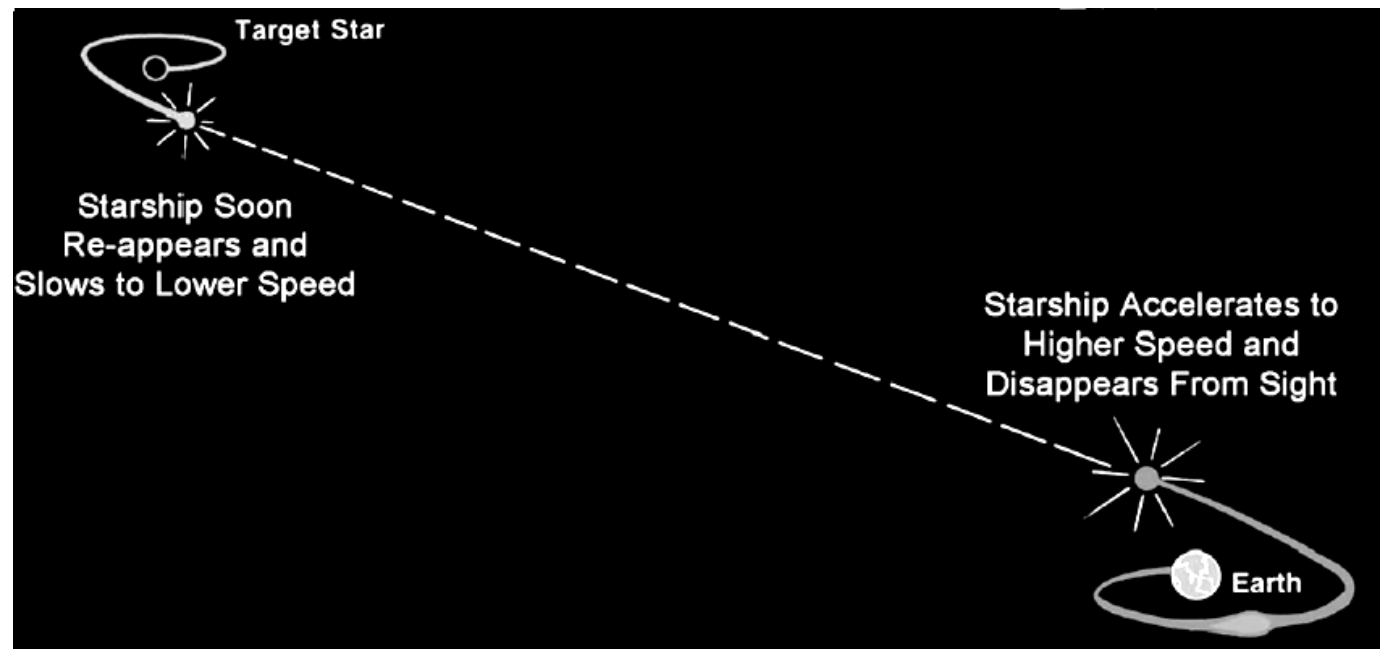

Figure 27. Interstellar Travel to the Star (H.D.Froning)

Starship accelerates away from the Earth, disappears and re-appears after Hyper-Space navigation. But during these Hyper-Space navigation of disappearance, the starship, in effect, leaps high above space-time and over stupendous distances to reach speeds that are substantively billions of times light-speed.

Starship flight can also be viewed from the perspective of an Earth observer who is watching a starship fly away - accelerating in the direction of its target (a planet in another solar system) and then vanishing from sight as its initial acceleration ends. The starship then re-appears after Hyper-Space navigation - at the speed it disappeared at. But the starship is now suddenly 400 light-years away - very near to its destination. By plunging into Hyper-Space featuring an imaginary time (i.e., Imaginary Time Hole), the starship detours the imaginary time tunnel (see Figure 22), apparently exceeds the speed of light. 
Finally, we compare the navigation features of the wormhole and the time-hole. Both navigation methods allow interstellar travel in a short period of time, but the features of the navigation, theoretical and technical issues are different.

Concerning the wormhole, regrettably, since the size of wormhole $\left(\sim 10^{-35} \mathrm{~m}\right)$ is smaller than the atom, and moreover the size is predicted to fluctuate theoretically due to instabilities, space flight through the wormhole is difficult technically and it is unknown where to go and how to return. Moreover, since the solution of wormhole includes a singularity, this navigation method theoretically includes fundamental problems: it is reported from numerical calculation that the wormhole solution considered by Thorn is an unstable solution.

It is premised on the existence of negative energy with high density (about the center of a neutron star) which is difficult with the current technology, and it is completely unknown how to go through the wormhole or where the exit is.

In contrast to this, as described above, a plunging into Hyper-Space characterized by imaginary time would make the interstellar travel possible in a short time. We may say that the present theoretical limitation of interstellar travel by Special Relativity (time paradox) is removed. The Hyper-Space navigation theory discussed above would allow a starship to start at any time and from any place for an interstellar travel to the farthest star systems, the whole mission time being within human lifetime (Figure 26, Figure 27).

\section{Conclusion}

The efforts of Robert Goddard and Von Braun's predecessors led to the successful landing of human beings on the moon and the exploration of the solar system planets by unmanned spacecraft. The development and achievements of the chemical rocket that supported it are great. Future developments such as ion thrusters, which are the subsequent electric propulsion, and hall thrusters under development, are desired.

On the other hand, this paper shows the possibility of various application development of the undeveloped laser thruster using water as a propellant.

However, since thrust and acceleration performance is insufficiency in order to challenge the solar system exploration and stellar system exploration, so a field propulsion of high acceleration that can be generated in a short time is necessary.

Also, there is no propulsion theory that exceeds the speed of light; even the field propulsion, its maximum theoretical speed is the quasi-light speed near the speed of light. For this reason, stellar system exploration as which the cruising range of a light-year unit is required needs not propulsion theory, but navigation theory such as worm holes and time holes. The realistic interstellar exploration can be possible by combining both a space drive propulsion theory with a hyper-space navigation theory (i.e., time-hole navigation). Hyper-Space navigation can be used at all times and everywhere in Real-Space without any restrictions to the navigation course.

\section{Acknowledgement}

The author thanks to Mr. Herman David Froning for providing the appropriate figures. 


\section{References}

Kuninaka, H. (2006). Powered Flight of HAYABUSA in Deep Space. https://doi.org/10.2514/ 6.2006-4318

Niino, M., Minami, Y., \& Nakamura, T. (1997). Study and Research Result of Advanced Space Propulsion Investigation Committee (ASPIC). 48th Congress of the International Astronautical Federation (IAF), (IAA-97-IAA.4.1.09).

Minami, Y., \& Uchida, S. (2013). Conceptual study of manned space transportation vehicle using laser thruster in combination with the H-II rocket. Acta Astronautica, 82. https://doi.org/10.1016/j.actaastro.2012.05.004

Minami, Y., \& Uchida, S. (2009). Water Vapour Propulsion Powered By A High-Power Laser-Diode. JBIS, 62.

Minami, Y., \& Uchida, S. (2015). Conceptual Design of Manned Space Transportation Vehicle (MSTV) Using Laser Thruster in Combination with H-II Rocket, presented at Satellite-2015 conference, August 17-19, 2015 Houston, Texas, USA.

Minami, Y., Uchida, S., \& Takahashi, Y. (2017). Small-Sized Laser Thruster for Attitude Control and Trajectory Control of Satellite. IOSR Journals: International Organization of Scientific Research (IOSR), 4(5) 44-56.

Minami, Y. (2015). An Introduction to Concepts of Field Propulsion. JBIS, 56, 350-359.

Minami, Y. (2015). Space propulsion physics toward galaxy exploration. J Aeronaut Aerospace Eng, 4(2). https://doi.org/10.4172/2168-9792.1000149

Minami, Y., \& Froning, H. D. (2017). Field Propulsion Physics and Intergalactic Exploration. Nova Science Publishers.

Minami, Y. (2019). New Development of Space Propulsion Theory -Breakthrough of Conventional Propulsion Technolog Y. International Journal of Advanced Engineering and Management Research, 4(1).

Minami, Y. (2005). A Perspective of Practical Interstellar Exploration: Using Field Propulsion and Hyper-Space Navigation Theory in the proceedings of Space Technology and Applications International Forum (STAIF-2005). https://doi.org/10.1063/1.1867273

Minami, Y. (2019). STAR FLIGHT THEORY: By the Physics of Field Propulsion. LAMBERT Academic Publishing.

Minami, Y. (1997). Spacefaring to the Farthest Shores-Theory and Technology of a Space Drive Propulsion System. Journal of the British Interplanetary Society (JBIS), 50, 263-276.

Minami, Y. (1998). Conceptual Design of Space Drive Propulsion System. STAIF-98, edited by Mohamed S. El-Genk, AIP Conference Proceedings 420, Part Three, 1516-1526, Jan.25-29, 1998, Albuquerque, NM, USA. https://doi.org/10.1063/1.54961

Minami, Y. (2014). A Journey to the Stars - By Means of Space Drive Propulsion and 
Time-Hole Navigation. LAMBERT Academic Publishing.

Forward, R. L. (1989). Space Warps: A Review of One Form of Propulsionless Transport. JBIS, 42, 533-542. https://doi.org/10.2514/6.1989-2332

Froning Jr, H. D. (1983). Requirements for Rapid Transport to the Further Stars. JBIS, 36, 227-230.

Minami, Y. (1993). Hyper-Space Navigation Hypothesis for Interstellar Exploration. 44th Congress of the International Astronautical Federation (IAF).

Minami, Y. (2003). Travelling to the Stars: Possibilities Given by a Spacetime Featuring Imaginary Time. JBIS, 56, 205-211.

Minami, Y. (2014). Interstellar travel through the Imaginary Time Hole. Journal of Space Exploration, 3, 206-212.

\section{Copyright Disclaimer}

Copyright for this article is retained by the author(s), with first publication rights granted to the journal.

This is an open-access article distributed under the terms and conditions of the Creative Commons Attribution license (http://creativecommons.org/licenses/by/3.0/). 